\title{
Optimization of Multiple Satisfaction Levels in Portfolio Decision Analysis
}

\author{
Maria Barbati ${ }^{1}$, Salvatore Greco ${ }^{1}$, , Miłosz Kadziński ${ }^{3}$, and Roman \\ Słowiński ${ }^{3,4}$
}

${ }^{1}$ University of Portsmouth, Portsmouth Business School, Centre of Operations Research and Logistics (CORL), Portsmouth, United Kingdom

${ }^{2}$ Department of Economics and Business, University of Catania, Catania, Italy

${ }^{3}$ Institute of Computing Science, Poznań University of Technology, Poznań, Poland

${ }^{4}$ Systems Research Institute, Polish Academy of Sciences, Warsaw, Poland

\begin{abstract}
We consider a portfolio decision problem in which a set of projects forming a portfolio has to be selected taking into account multiple evaluation criteria and some constraints related to the limited resources (e.g., available budget). Traditionally, such a problem has been approached by Multiple Attribute Value Theory (MAVT) with the aim of maximizing the sum of values associated with the projects included in the selected portfolio. Using MAVT, one represents preferences on the individual projects, and a value of a portfolio is just an aggregate of values of the component projects. This linear value approach does not explicitly account for portfolio balance requirements, raising the risk of selecting a portfolio which is, e.g., composed of projects with good evaluations on the same criterion or on the same small subset of criteria. Thus, we propose a different approach that enables the Decision Maker (DM) to control the distribution of good evaluations on different criteria over the projects composing a portfolio. With this aim, for each criterion we fix a certain number of reference levels corresponding to the qualitative satisfaction degrees. The number of
\end{abstract}


projects entering a portfolio and attaining each of these levels becomes an objective to be maximized. To solve thus formulated multi-objective optimization problem, we use Dominance-based Rough Set Approach (DRSA). The DM is expected to point out some prospective portfolios in a current sample of non-dominated portfolios. DRSA represents the DM's preferences with a set of decision rules induced from such indirect preference information. Their use permits to progressively focus the search on the part of the non-dominated portfolios that satisfy the DM's preferences in the best way.

Keywords: Portfolio Decision Analysis, Multiple Criteria Decision Aiding, Dominance-based Rough Set Approach, Interactive Multiobjctive Optimization, Satisfaction levels

\section{Introduction}

Portfolio decision problem concerns selecting a subset of projects, called portfolio, that optimizes some objectives while respecting several constraints. Formally, portfolio selection is formulated as a multiple objective optimization problem where each project is evaluated on multiple criteria and has an associated cost [65]. In this perspective, one optimizes the performance of projects contained in the selected portfolio so that the comprehensive cost of such a portfolio does not exceed the available budget. The dedicated solution methods for thus formulated problem need to combine problem structuring, preference elicitation, multiple criteria methods for assessment of alternative portfolios, optimization algorithms, and interactive software engaging stakeholders.

Portfolio decision problems are typical for numerous real-life applications. For example, firms seek to attain their goals by allocating resources to a subset of projects with the aim of generating growing profits or increasing productivity $[58,62]$. Furthermore, public organizations allocate funds to initiatives delivering some services to the citizens (e.g., a public library to its clients [59]), decide upon funding processes [32] and research and development (R\&D) projects [40], or choose the sites to dispose waste [41]. Finally, manufacturing plants deal with the configuration of their product lines in order to decide which products to make [71].

The body of theory, methods, and practice that deals with portfolio decision problems has been called Portfolio Decision Analysis (PDA) [65]. PDA aims at supporting the DMs in solving mathematical modeling that accounts for the relevant constraints, preferences, and uncertainties. The current challenges in PDA concern the formalization of the elicitation of DM's preferences and the systematization of the selection process. According to [65], in the next years PDA will face further challenges: from the institutionalization in the organizational decision making to the advances in information technology (IT) and software tools. Among them, we also find the formulation of relevant objectives and development of solution methods to be incorporated into PDA processes.

Many methodologies have been proposed to approach the portfolio optimizations problems. Selecting the best solution is not a trivial task. To simplify it, one often represents the DM's preferences with a value 
function that makes projects and portfolios more comparable. In general, given a set of feasible projects evaluated on a set of criteria, the overall value of a portfolio is formulated with a linear value approach, as the sum of values associated with all individual projects included in the portfolio. Then, the problem is solved by maximizing thus defined value function of a portfolio. Alternatively, one can define for each criterion an ideal value. In this case, the problem consists in choosing the portfolio that minimizes some aggregated weighted difference of the performances on individual criteria from the ideal values. In this sense, many examples have been defined (see, e.g., [6]) in different fields such as, e.g., information systems, transportation [73], public investment [78, 79], forest management problems [2, 21, 44], or facility location problems [18].

In particular, if each item (project) had several profit values and an associated weight (cost), the problem can be formulated in terms of a multi-objective knapsack problem. The profit values of items entering a knapsack (portfolio) are aggregated by some objective functions (evaluation criteria) to be optimized. To solve the problem, one has to find a knapsack that optimizes the objective functions while keeping the sum of item weights within the limited knapsack capacity. Several algorithms have been defined for such a problem with the aim of computing a good approximation of the set of solutions covering all possible trade-offs between the different objectives. These algorithms can be divided into exact approaches (see, e.g., [7], [43], and [64]), heuristics or metaheuristics (e.g., [22], [33], [74], and [76]), and combinations of these (see, e.g., [12], [14], [15], [54], and [72]). For a complete review of these algorithms, see [19].

When portfolio decision problem is modeled as a multi-objective knapsack problem, many simplifications are made. These include assuming a priori knowledge on the exact consequences of selecting each project, using deterministic data, or admitting additive objective values and costs. As noted in [11], many modifications to this approach should be made so that it is suitable for dealing with a broader class of real-world problems. Moreover, even if we knew all possible solutions, we are still far from selecting a single one that would best fit the DM's preferences. Indeed, the assumption that the portfolios with higher values are preferred by the DM [65], does not permit to take into account a distribution of good performances on the whole set of considered criteria. This, in turn, can bring to situations where one selects a portfolio of projects having very good evaluations on the same single criterion and bad evaluations on the remaining criteria. Additionally, if targets were set from the beginning for each criterion, the learning process derived from constructing the portfolios through an interactive procedure is not possible. Even the aggregation of the differences between the targets and the values of each criterion should evolve during the interaction with the DM.

Our study addresses these challenges by providing a new formulation of a portfolio decision problem that involves some qualitative satisfaction levels defined on each evaluation criterion referring to a single project. It assumes that a desirable portfolio should maximize the numbers of component projects that attain particular satisfaction levels, thus, replacing the sum of marginal values of component projects by a vector of the numbers of projects attaining particular satisfaction levels. This permits better perception of the quality of different portfolios by the DM.

Let us justify our approach through the following didactic example. The manager of a football club wants 
to select 16 players for his team including 11 regular ones and 5 substitutes. Suppose that maximizing the sum of the values associated with the individual players, 16 goalkeepers are deemed as the 16 best football players, and that the available budget is sufficient to recruit all of them. Clearly, a football team needs to involve defenders, midfielders, and forwards. Therefore, there is a necessity to take into consideration a proper distribution of good evaluations among all considered criteria. However, this point, being of primary relevance in PDA, is not explicitly considered when applying MAVT. In our proposal, it is handled by maximizing the number of projects that attain some pre-defined levels of quality on different considered criteria. In this way, one avoids the concentration of good performances on one or a small subset of criteria. Instead, the research is focused on portfolios with projects having good evaluations well distributed on all considered criteria or a subset of criteria desired by the DM. In terms of the above example, the manager would ensure that a certain number of players would be good with respect to ball handling, positioning and quick reflexes, being typical criteria for goalkeepers, but he would also maximize the number of players being good with respect to passing/receiving, dribbling, shooting, tackling, trapping, sprinting, endurance, heading, and juggling. In this way, the team could be finally composed of a well distributed number of defenders, midfielders, and forwards, apart from the two or three goalkeepers.

Indeed, if the aim is to select a portfolio such that for each criterion there is a certain number of projects with good performances, the problem is to define the underlying thresholds, possibly considering different levels of quality. We propose to use for this an Interactive Multi-objective Optimization (IMO) (for an updated survey, see [57] and [56]) by decision rules. These are induced from the DM's preference information by the Dominance-based Rough Set Approach (DRSA) [25]. This new approach is called IMO-DRSA [26].

The IMO-DRSA approach [26] fits well this purpose, because it permits to take into account constraints on the minimal number of projects having performances that meet different satisfaction levels on the considered criteria. These constraints are expressed in terms of "if ..., then ..." decision rules, such as "if a portfolio includes at least 4 projects which are at least medium with respect to criterion $g_{1}$ and at least 6 projects that are at least moderately good on criterion $g_{2}$, then the portfolio is good".

These rules are induced from some preference information supplied by the DM who is asked to indicate some projects as "relatively good" among a small set of projects that are presented to her/him at a certain stage of the decision aiding procedure. More precisely, the decision rules show some minimal conditions that permit to explain the judgment of the DM, permitting to distinguish "relatively good projects" from the others. In fact, each decision rule describes a set of projects (possibly even a singleton) that support the rule and have been judged as "relatively good" by the DM. On the other hand, the projects that have not been deemed as "relatively good" fail to satisfy these conditions in the antecedent of the rule. Therefore, the decision rules represent synthetic explanations of the DM's judgments expressed in simple and understandable terms (so that no specific technical background is required from her/him). Thus, for the DM it is very natural to discuss and possibly criticize the rules, taking into account in a transparent way the specific judgments that originated the rules. Moreover, the clear language of the rules permits the DM to select with conviction those rules which are more meaningful to her/him, in the sense that they represent her/his preferences in the best way. These are advantages of IMO-DRSA procedures, and, in general, of 
DRSA.

However, application of the IMO-DRSA approach in a specific context of a portfolio decision problem brings even more benefits. In fact, the syntax of the rules is based on the idea that a good portfolio should contain a minimal number of projects attaining some quality levels on some relevant criteria. Thus, the IMO-DRSA procedure we are proposing permits to fix these thresholds in a way that is transparent and convincing for the DM. Moreover, the final solution is found by a process of progressive inclusion of more and more demanding thresholds that gradually constrain the set of portfolios satisfactory these requirements. For the DM, this involves a learning process that allows her/him to learn which types of thresholds represent better her/his preferences and which are the portfolios reaching these thresholds. The process stops when the DM acquires a sufficient comprehension of the problem in terms of the thresholds (s)he can aspire and those (s)he would like to attain, and, in view of this, when (s)he identifies a solution that is satisfactory for her/him. In simple words, the IMO-DRSA procedure permits the DM to look at the decision process in the right perspective (attainment of thresholds in terms of the number of projects that meet particular quality levels on the considered criteria) and permits to follow this direction until a decision is matured in terms of conviction about a satisfactory solution.

The paper is organized in the following way. In Section 2, we review the PDA processes that have been proposed in the literature. Section 3 presents both the new formulation for portfolio decision problems and the adopted solution method. In Section 4, we recall the main features of DRSA, and discuss how it can be adapted to PDA. Section 5 illustrates its use on a didactic example, while Section 6 concludes the paper.

\section{A review of selected solution methods for Portfolio Decision Problems}

In this section, we review the methods that have been proposed in the literature for handling PDA problems. Many of these approaches consist of several stages that are related to different phases of selecting a portfolio. As indicated in [4], portfolio selection process can be divided into three phases: strategic considerations, evaluation of individual projects, and portfolio selection. Each of them can be again divided in finer stages, and it is possible to incorporate different methodologies for each of them.

In PDA approaches, Multiple Criteria Decision Analysis (MCDA) methods often play a vital role when selecting the best portfolio or ranking different portfolios from the best to the worst [24]. For example, the PROMETHEE V method [75] employs the concepts of boundary portfolios which are maximal in the sense that they cannot accommodate more projects without violating resource constraints, and $c$-optimal portfolios that are deemed as optimal when considering only $c$ projects. Furthermore, [1] applied some MCDA methods for selecting a portfolio of information system projects considering strategic, technical, and organizational criteria. Moreover, [80] developed a three-step process, in which Analytic Network Process was used to evaluate influence, power, and performance scores of suppliers in a green supply chain. To support the analysis of the alternative scenarios for the local energy planning, [51] developed a multiple criteria ordinal regression approach, whereas the problem of selecting some refinery upgrade projects was addressed in [45] with a dedicated stochastic algorithm. Afterwards, [31] proposed a hybrid methodology 
that integrates the Analytic Hierarchy Process (AHP) with a binary integer portfolio optimization model. The AHP component allows the DMs to incorporate qualitative and intangible criteria into the objective function of the optimization model. Finally, [77] integrated AHP with Data Envelopment Analysis to select R\&D projects under the optimisation of five criteria.

The vast majority of PDA approaches incorporate interaction with the DM. The importance of interactive procedures has been raised in [4]. In particular, it has been emphasized how the solutions provided by the algorithms or models should be checked by the DM. Additionally, the DMs should provide feedback on the selected portfolio and on the consequences of changes for the projects introduced in it [69]. The actual preferences of the DM are captured and made explicit by a Decision Support System (DSS) which provides an interface for interaction throughout the different stages. This allows to avoid a priori formulation of the DM's preferences, and motivates the successful implementation of the selected projects [23].

An example of a DSS incorporating a two-phase solution process has been proposed in [23]. The first phase is used to aggregate the multiple objectives into a single objective function according to some selected MCDA method. In the other phase, one solves a binary optimization model with the derived single objective function. The obtained solutions are presented to the DM in a portfolio matrix, displaying time to complete and risk associated with the project. In this regard, they are used as a starting point to reach satisfactory portfolios through the following interactions with the DSS.

In the same spirit, [69] described a three-phase approach, embedded in a DSS, to assist the DM in constructing the most attractive (project) portfolio. After a screening procedure, a multi-objective integer linear programming model is employed. It accounts for the overall benefits and projects interdependencies with logical and strategic requirements, as well as for the resource and benefit constraints. The efficient portfolios are determined through enumeration. The DM can explore the solution space starting from one of the efficient portfolios and iteratively setting or modifying aspiration levels for the objectives. The search procedure is continued with the analysis of different portfolios until the DM retains the most satisfactory one.

Furthermore, [39] proposed to divide the process of choosing a portfolio (of weapon systems) in two stages. The first stage is oriented towards identification of some efficient solutions, whereas the other stage involves the use of an algorithm that compares the performances of several weapon systems. The elicited preferences are used to determine precise values for the criteria weights.

Conversely, [47] dealt with the presence of incomplete information. This increased the number of efficient portfolios and made it more difficult to enumerate all non-dominated ones in case many projects are considered. To approach the problem, they introduced a dynamic programming algorithm. The DM could identify the most preferred portfolio with a robustness measure. Subsequently, [48] extended this approach considering interdependencies between projects, incomplete cost information, and variable available capital.

Also [49] proposed a DSS, called PROBE, that integrates two main components: an MCDA method that solves a MKP based on uncertain values and cost associated to each project, and a PDA method that checks the robustness of portfolio comparing it to another portfolio and evaluating the regret of the DM in case of choosing a less favored one. Later, [50] used PROBE to evaluate the investments projects of a 
leading company in the energy sector. A DSS adopting interacting visualisation charts and, alternatively, a linear single criterion optimisation model is described in [16]. The underlying software provides timeline, cost, and risk charts that help the DMs to visualise different scenarios determined by the choice of projects to be implemented.

The interactive methods are used also for determining the criteria weights. Some of these approaches achieve this through an initial interaction with the DM (see, e.g., [39] and [47]). Furthermore, [5] presented a new formulation for PDA, integrating the decision variable space and the space of criteria weights. In this way, efficient portfolios and associated weights can be identified simultaneously. Finally, [46] developed a more general class of portfolio value functions which deploy symmetric multi-linear functions to capture nonlinearities in the criterion specific portfolio values. These per-criterion portfolio values can be aggregated with some additive, multiplicative or multi-linear functions allowing a rich representation of preferences. In what follows, we present a methodology that avoids the specification of criteria weights.

\section{The proposed model and methodology for Portfolio Decision Problems}

The considered portfolio decision problem concerns a set of projects $A=\left\{a_{1}, \ldots, a_{j}, \ldots, a_{n}\right\}$. Each project $a_{j} \in A$ is evaluated on a set of criteria $G=\left\{g_{1}, \ldots, g_{i}, \ldots, g_{h}\right\}$ and has an associated cost $c_{j} \in \mathbb{R}^{+}$. The available budget is denoted by $C$.

For the sake of simplicity, without the loss of generality, suppose that all criteria $g_{i} \in G$ are of a gain type, such that the greater $g_{i}\left(a_{j}\right)$ the better the evaluation of project $a_{j} \in A$ on criterion $g_{i}$. For each $g_{i} \in G$, the DM needs to fix a set $L_{i}$ consisting of $G(i)$ quality thresholds

$$
L_{i}=\left\{l_{1, i} ; \ldots ; l_{G(i), i}: l_{1, i}<l_{2, i}<\ldots<l_{G(i), i}\right\}
$$

permitting to define a set $\mathcal{C}_{i}$ consisting of $G(i)+1$ qualitative satisfaction classes $C_{t, i}$

$$
\mathcal{C}_{i}=\left\{C_{1, i}, \ldots, C_{G(i)+1, i}\right\}
$$

such that the greater $t=1, \ldots, G(i)+1$, the more preferred is the project from class $C_{t, i}$. The projects $a_{j} \in A$ are assigned to satisfaction classes $C_{t, i} \in \mathcal{C}_{i}$ according to the following rule: for all $a_{j} \in A$

- $a_{j}$ is assigned to class $C_{1, i}$ if $g_{i}\left(a_{j}\right)<l_{1, i}$

- $a_{j}$ is assigned to class $C_{t, i}$ if $l_{t-1, i} \leq g_{i}\left(a_{j}\right)<l_{t, i}$;

- $a_{j}$ is assigned to class $C_{G(i)+1, i}$ if $l_{G(i), i} \leq g_{i}\left(a_{j}\right)$.

For example, when considering criterion $g_{i}$ with a numerical evaluation scale $[0,100]$, one can fix a set $L_{i}=\{15,45,80\}$ of $G(i)=3$ quality thresholds to define four satisfaction classes: "weakly satisfactory", "satisfactory", "very satisfactory", and "extremely satisfactory". Then, for any project $a_{j} \in A$ we have that with respect to criterion $g_{i}$ : 
- $a_{j}$ is "weakly satisfactory" if $g_{i}\left(a_{j}\right)<15$;

- $a_{j}$ is "satisfactory" if $15 \leq g_{i}\left(a_{j}\right)<45$;

- $a_{j}$ is "very satisfactory" if $45 \leq g_{i}\left(a_{j}\right)<80$;

- $a_{j}$ is "extremely satisfactory" if $80 \leq g_{i}\left(a_{j}\right)$.

Let us remark that even if the definition of the satisfaction thresholds $l_{t, i}$ can be demanding for the DM, this can significantly enhance the quality of the whole decision process, because it permits to translate the results of the multi-objective optimization in well understandable terms involving the quality thresholds that are meaningful for the DM. Moreover, during the decision process (s)he can understand better which are the quality thresholds to which (s)he is sensitive, and even change their values or the number of thresholds $l_{t, i}$. The meaningfulness of the thresholds $l_{t, i}$ can change also because during the decision process the DM learns about the set of feasible solutions. For example, if for one criterion it is easier than expected for the projects to attain the highest quality levels (e.g., when the majority of projects are assigned to the most satisfactory class), the thresholds can be modified to be more demanding. In the opposite case, if for one criterion it is more difficult than expected to find highly satisfactory projects, the thresholds can be relaxed. In this way, the definition of the thresholds offers another possibility of interaction with the DM within the decision process.

The thresholds $l_{t, i}$ are used as follows in an interactive multi-objective optimization procedure. First, observe that each subset of projects $P \subseteq A$ is a potential portfolio that can be identified with a vector $\mathbf{x}=\left[x_{1}, \ldots, x_{j}, \ldots, x_{n}\right]$, such that $\forall a_{j} \in A$ :

$$
x_{j}= \begin{cases}1, & \text { if } a_{j} \in A \text { is contained in } P \\ 0, & \text { otherwise }\end{cases}
$$

For each portfolio $P \subseteq A$, each criterion $g_{i} \in G$ and each satisfaction level $l_{t, i} \in L_{i}$, we consider the set of projects attaining threshold $l_{t, i}$ :

$$
P_{t, i}=\left\{a_{j} \in P: g_{i}\left(a_{j}\right) \geq l_{t, i}\right\} .
$$

For the sake of simplicity, in what follows we shall write $\left|P_{t, i}\right|$, as $F_{t, i}(\mathbf{x})$ to refer to portfolio $P$ identified by vector $\mathbf{x}, g_{i} \in G$ and $l_{t, i} \in L_{i}$.

Taking into account all above elements, we formulate the portfolio decision problem as the following multi-objective optimization problem:

$$
\max F_{t, i}, \forall g_{i} \in G, \forall l_{t, i} \in L_{i}
$$

subject to:

$$
\sum_{a_{j} \in A} c_{j} x_{j} \leq C
$$


We can reformulate the objectives $F_{t, i}$ of the above problem in terms of the binary variables $x_{j}$ as follows. Let us introduce an indicator for each project $a_{j} \in A$, criterion $g_{i} \in G$, and satisfaction level $l_{t, i} \in L_{i}$ :

$$
h_{i, j, t}= \begin{cases}1, & g_{i}\left(a_{j}\right) \geq l_{t, i} \\ 0, & \text { otherwise }\end{cases}
$$

so that for portfolio $P$ identified by vector $\mathbf{x}=\left[x_{1}, \ldots, x_{j}, \ldots, x_{n}\right]$, we get

$$
F_{t, i}=\sum_{a_{j} \in A} h_{i, j, t} x_{j}
$$

Then, the above multi-objective optimization problem can be formulated as follows:

$$
\max \sum_{a_{j} \in A} h_{i, j, t} x_{j}, \quad \forall g_{i} \in G, \quad \forall l_{t, i} \in L_{i}
$$

subject to

$$
\sum_{a_{j} \in A} c_{j} x_{j} \leq C
$$

Clearly, the above problem is a multi-objective 0-1 linear programming optimization problem with $\sum_{i=1}^{h} G(i)$ objectives. That is, the number of objectives is equal to the number of satisfaction levels defined for all criteria, and the objectives are the number of projects attaining the quality thresholds $l_{t, i} \in L_{i}$ on $g_{i} \in G$.

Several algorithms, mainly exact, have been provided in the literature to find solutions to multi-objective 0-1 linear programming problems (for a review, see [20]). When dealing with small problem instances, some algorithms can look for the whole set of efficient solutions. These include the branch and bound algorithms [42] or the $\epsilon$ constraint method $[13,55]$. Some interactive algorithms integrate optimization procedures $[5,3,53]$ with the aim of singling out the set (possibly a singleton) of the most preferred solutions for the DM. In the same perspective, other methods suggest the adoption of a linear value approach (see, e.g., [65]) or the use of a goal programming procedure [63]. In what follows, we provide a small example that highlights why these two approaches may not lead to appropriate solutions from the viewpoint of concerns underlying the multiple satisfaction level optimization based on the above defined objectives $F_{t, i}$.

Example. Let us consider five projects evaluated on three gain-type criteria and having an associated cost (see Table 1). For each criterion, we define one satisfaction level equal to 200. That is, a project is satisfactory with respect to a given criterion if its evaluation is not smaller than 200, and unsatisfactory, otherwise. The available budget is equal to 100 .

Given the budget constraint, the portfolios being potential solutions of the multi-objective problem are composed of any three projects. Thus, we have a set $\mathbf{P}=\left\{P_{1}, \ldots, P_{10}\right\}$ of ten feasible portfolios (see Table 2 ). The linear value approach maximizes the value function of a portfolio $P$ defined as: 
Tab. 1: Evaluations on three gain-type criteria and the underlying costs for five projects considered in the example.

\begin{tabular}{|c||c|c|c|c|}
\hline Projects & $g_{1}$ & $g_{2}$ & $g_{3}$ & Cost \\
\hline \hline$a_{1}$ & 10000 & 10 & 10 & 30 \\
$a_{2}$ & 10000 & 46 & 38 & 30 \\
$a_{3}$ & 400 & 400 & 480 & 30 \\
$a_{4}$ & 400 & 400 & 400 & 30 \\
$a_{5}$ & 800 & 250 & 250 & 30 \\
\hline
\end{tabular}

Tab. 2: Feasible portfolios composed of projects presented in Table 1.

\begin{tabular}{|c|c|c|c|c|c|c|}
\hline Portfolio $(P)$ & Projects in $P$ & $U(P)$ & $\Delta(P)$ & $F_{1,1}$ & $F_{1,2}$ & $F_{1,3}$ \\
\hline$P 1$ & $a_{1} a_{2} a_{3}$ & 21384 & 0.57 & 3 & 1 & 1 \\
\hline$P 2$ & $a_{2} a_{3} a_{4}$ & 12564 & 0.48 & 3 & 2 & 2 \\
\hline$P 3$ & $\begin{array}{lll}a_{3} & a_{4} & a_{5}\end{array}$ & 3780 & 0.92 & 3 & 3 & 3 \\
\hline$P 4$ & $a_{1} a_{4} a_{5}$ & 12520 & 0.46 & 3 & 2 & 2 \\
\hline$P 5$ & $a_{2} a_{4} a_{5}$ & 12584 & 0.46 & 3 & 2 & 2 \\
\hline$P 6$ & $a_{1} a_{3} a_{4}$ & 12500 & 0.48 & 3 & 2 & 2 \\
\hline$P 7$ & $a_{1} a_{2} a_{5}$ & 21404 & 0.74 & 3 & 1 & 1 \\
\hline P8 & $a_{1} a_{2} a_{4}$ & 21304 & 0.60 & 3 & 1 & 1 \\
\hline$P 9$ & $a_{2} a_{3} a_{5}$ & 12664 & 0.46 & 3 & 2 & 2 \\
\hline$P 10$ & $a_{1} a_{3} a_{5}$ & 12600 & 0.46 & 3 & 2 & 2 \\
\hline
\end{tabular}

$$
U(P)=\sum_{a_{j} \in P} U\left(a_{j}\right)=\sum_{a_{j} \in P} \sum_{g_{i} \in G} w_{i} g_{i}\left(a_{j}\right)=\sum_{a_{j} \in A} \sum_{g_{i} \in G} w_{i} g_{i}\left(a_{j}\right) x_{j}^{P},
$$

with $\mathbf{x}^{P}=\left[x_{1}^{P}, \ldots, x_{5}^{P}\right]$ being the vector characterizing portfolio $P$, and $w_{i}$ representing a non-negative weight for criterion $g_{i}$. In what follows, for the sake of simplicity, we assume weights equal to one for all criteria.

We also test a compromise programming approach [63] for which the aim is to minimize the maximum deviation from the ideal point, i.e., the point with the best evaluation $g_{i}^{*}=\max _{\mathbf{P}} \sum_{a_{j} \in P} g_{i}\left(a_{j}\right)$ that a feasible portfolio $P$ can attain on all criteria $g_{i} \in G$. Different metrics can be adopted in order to define the closeness of the obtained portfolio to the ideal point. Following [18], in order to get a balanced solution, we minimize the maximum deviation $\Delta_{i}$, on the set of criteria $g_{i} \in G$, defined as

$$
\Delta_{i}=\frac{g_{i}^{*}-\sum_{a_{j} \in P} g_{i}\left(a_{j}\right)}{g_{i}^{*}} .
$$

Then, the distance of portfolio $P \in \mathbf{P}$ from the ideal point is $\Delta(P)=\max _{g_{i} \in G} \Delta_{i}$.

In Table 2, we report the feasible portfolios, the projects included in each portfolio, the corresponding value functions $U(P)$, the distances $\Delta(P)$, and the values $F_{1, i}$ for $g_{i}, i=1,2,3$, indicating the number of 
satisfactory projects in terms of a particular criterion in the portfolio. On one hand, the optimal portfolio with respect to the linear value approach $U$ is $P 7$, containing projects $a_{1}, a_{2}$, and $a_{5}$. On the other hand, there are four portfolios optimal with respect to the compromise programming procedure being $P 4, P 5, P 9$, and $P 10$. Let us observe that if one would like to maximize the number of satisfactory projects with respect to the considered criteria $F_{1, i}, g_{i} \in G$, as proposed in our approach, then one should observe that portfolio $P 3$ with 3 satisfactory projects on each criterion is dominating all other portfolios from $\mathbf{P}$. This portfolio is not among the preferred ones suggested by the linear value approach or by the compromise programming procedure.

Let us observe that the multiple satisfaction levels we propose to consider, can be applied also in case of fractionable projects. Then, variables $x_{j}, j=1, \ldots, n$, are no more binary, but instead represent a portion of a resource allocated to a given option (e.g., an extension of land devoted to some culture in forest management [44]).

Let us also remark that any multi-objective optimization procedure can be applied to search for portfolios being the most preferred with respect to the number of projects attaining particular satisfaction levels on each criterion. In the next section, we propose to apply a procedure called DRSA-IMO that permits to consider the satisfaction levels as an inherent part of the resolution process.

\section{Dominance-based Rough Set Approach for Interactive Multi-objective Optimization in Portfolio Decision Analysis}

To handle the problem introduced in the previous section, we apply DRSA-IMO in the alternating phases of computation and dialog with the DM.

Computation phase. In the computation phase, a set of Pareto-optimal solutions of the considered multiobjective optimization problem are computed and proposed to the DM. This can be done in different ways. In general, it is advisable that the DM participates in the construction of such solutions by fixing the minimal values for some of the considered objectives and searching for the solutions optimizing some of the other objectives, with a final check that the obtained solution is Pareto-optimal.

A simpler approach can relieve the DM from the active participation in the computation phase. In this spirit, one can generate the set of solutions to be presented to the DM by optimizing each single objective function in (7), while ensuring that the generated solution is Pareto-optimal. In case of our formulation of the portfolio decision problem, this procedure can be implemented as follows.

For each criterion $g_{i} \in G$ and for each satisfaction level $l_{t, i}, t=1, \ldots, G(i)$, one determines a portfolio that maximizes $F_{t, i}$, i.e., the number of projects that attain value at least $l_{t, i}$ on $g_{i}$. Then, for each pair 
$\left(t^{*}, i^{*}\right), l_{t^{*}, i^{*}} \in L_{i^{*}}, g_{i^{*}} \in G$, we determine the solution $\mathbf{x}_{t^{*}, i^{*}}$ that maximizes the sum of objective values:

$$
\sum_{l_{t, i} \in L_{i}, g_{i} \in G} F_{t, i}(\mathbf{x})
$$

under the constraint (8) of the original problem plus $F_{t^{*}, i^{*}}(\mathbf{x}) \geq F_{t^{*}, i^{*}}\left(\mathbf{x}_{t^{*}, i^{*}}\right)$. The latter constraint guarantees that the value of the previously optimized objective $F_{t^{*}, i^{*}}$ is not deteriorated.

In this way, in the computation phase, we find $\sum_{i=1}^{h} G(i)$ (not necessarily distinct) weakly non-dominated portfolios that maximize individually each objective $F_{t, i}$ and are guaranteed to be Pareto-optimal. This set of portfolios is proposed to the DM. The sets presented to the DM in the following iterations of the interactive procedure can be determined analogously.

Dialog phase. When a sufficient number of Pareto-optimal solutions have been found, in the dialog phase the DM can start her/his reflection to conclude that:

- among the proposed solutions there is one that can be selected, stopping the interactive procedure,

- or there is no satisfactory solution in the current problem setting, and pass to reformulation of the portfolio decision problem,

- or, if the DM is not completely satisfied with some of the presented solutions, to criticize the portfolios by classifying them into "good" and "others".

In the last scenario, the provided classification data is structured using DRSA with the aim of inducing a set of certain decision rules covering the portfolios belonging to the lower approximation of class "good". This set of rules is representing preferences of the DM. Once the DM selects the rule that best reflects her/his current aspiration, the elementary conditions of the selected rule are incorporated into the constraints of the multi-objective optimization problem to calculate a new sample of portfolios in the next computation phase, with the intention to better fit the DM's preferences.

\subsection{Dominance-based Rough Set Approach}

In this section we present the DRSA methodology which, starting from the preferences information supplied by the DM, permits to induce the decision rules that guide the interactive multiobjective optimisation process considered in our PDA approach.

Rough Set Theory [61] has shown to be very effective in the analysis of ambiguous and inconsistent data. In order to extend it to the preference ordered data appearing in the majority of real-world decision problems, Dominance-based Rough Set Approach (DRSA) [25] has been proposed (see, e.g., [29, 66, 67]). In what follows, we consider an ordinal classification (sorting) problem involving:

- a finite set of portfolios $\mathbf{X}=\left\{\mathbf{x}_{1}, \ldots, \mathbf{x}_{j}, \ldots, \mathbf{x}_{n}\right\}$; 
- a finite set of criteria $F=\left\{F_{1}, \ldots, F_{i}, \ldots, F_{m}\right\}$, with $F_{i}: \mathbf{X} \rightarrow \mathbb{R}$ measuring the quality of $\mathbf{x}_{i} \in \mathbf{X}$ with respect to $F_{i} \in F, i=1, \ldots, m$; for all portfolios $\mathbf{x}_{j} \in \mathbf{X}$, and for $F_{i} \in F$

$$
F_{i}\left(\mathbf{x}_{j}\right) \geq F_{i}\left(\mathbf{x}_{k}\right) \Leftrightarrow \mathbf{x}_{j} \succsim_{i} \mathbf{x}_{k}
$$

where $\succsim_{i}$ is the marginal preference related to criterion $F_{i} \in F$ and $\mathbf{x}_{j} \succsim_{i} \mathbf{x}_{k}$ reads " $\mathbf{x}_{j}$ is at least as good as $\mathbf{x}_{k}$ with respect to $F_{i}$ "; observe that $\succsim_{i}$ is a complete preorder, i.e., a strongly complete and transitive binary relation, defined on $F$;

- a finite set of preference ordered decision classes $\boldsymbol{C l}=\left\{C l_{1}, \ldots, C l_{z}\right\}$, such that for all $r, s=1, \ldots, z$, if $r>s$, then the portfolios from $C l_{r}$ are preferred to the portfolios from $C l_{s}$.

In this perspective let us remind that portfolio $\mathbf{x}_{j} Q$-dominates portfolio $\mathbf{x}_{k}$ with respect to $Q \subseteq F$ (shortly, $\mathbf{x}_{j}$ Q-dominates $\left.\mathbf{x}_{k}\right)$, denoted by $\mathbf{x}_{j} D_{Q} \mathbf{x}_{k}$ if $\mathbf{x}_{j}$ is at least as good as $\mathbf{x}_{k}$ for every criterion $F_{i} \in Q, F_{i}\left(\mathbf{x}_{j}\right) \geq$ $F_{i}\left(\mathbf{x}_{k}\right)$.

The interactive multi-objective procedure we present in the next sub-section, involves in each iteration a different set $\mathbf{X}$ of portfolios evaluated on criteria from set $F$. The DM is supposed to provide preference information in terms of classification performed on $\mathbf{X}$. Precisely, the DM indicates some prospective portfolios from $\mathbf{X}$ that distinguish positively from the others. In other words, the DM makes a binary ordinal classification of X into classes "good" and "others". DRSA permits to structure the provided preference information into lower and upper approximations of the decision classes, and then to induce "if ..., then ..." decision rules from these approximations, referring to the evaluations of portfolios on some selected criteria.

In case of binary ordinal classification, the basic concepts of DRSA are the lower and upper approximations of each class with respect to set of criteria $Q \subseteq F$. The lower approximation of a class contains all portfolios from set $\mathbf{X}$ that are consistent with the dominance principle, i.e., such that there is no case where a portfolio dominating another portfolio on criteria from set $Q$ would be assigned to a worse class, nor a scenario in which a portfolio dominated by another portfolio on criteria from set $Q$ would be assigned to a better class. The upper approximation of a class may contain inconsistent portfolios.

Formally, for each subset of criteria $Q \subseteq F$ and for each portfolio $\mathbf{x}_{j} \in \mathbf{X}$, one can define:

- a set of portfolios dominating $\mathbf{x}_{j}$, called Q-dominating set, $D_{Q}^{+}\left(\mathbf{x}_{j}\right)=\left\{\mathbf{x}_{k} \in \mathbf{X}: \mathbf{x}_{k} D_{Q} \mathbf{x}_{j}\right\}$;

- a set of portfolios dominated by $\mathbf{x}_{j}$, called Q-dominated set, $D_{Q}^{-}\left(\mathbf{x}_{j}\right)=\left\{\mathbf{x}_{k} \in \mathbf{X}: \mathbf{x}_{j} D_{Q} \mathbf{x}_{k}\right\}$.

Consequently, the lower approximation of class $C l_{\text {good }}$, denoted with $\underline{Q}\left(C l_{\text {good }}\right)$, is composed of portfolios $\mathbf{x}_{j} \in C l_{\text {good }}$ which are not dominated by any portfolio belonging to class $C l_{\text {others }}$. The upper approximation of class $C l_{\text {good }}$, denoted with $\bar{Q}\left(C l_{\text {good }}\right)$, is composed of all portfolios which dominate any portfolio $\mathbf{x}_{j} \in$ $C l_{\text {good }}$. Precisely, these approximations are defined as follows:

$$
\begin{aligned}
\underline{Q}\left(C l_{\text {good }}\right) & =\left\{\mathbf{x}_{j} \in \mathbf{X}: D_{Q}^{+}\left(\mathbf{x}_{j}\right) \subseteq C l_{\text {good }}\right\} \\
\bar{Q}\left(C l_{\text {good }}\right) & =\left\{\mathbf{x}_{j} \in \mathbf{X}: D_{Q}^{-}\left(\mathbf{x}_{j}\right) \cap C l_{\text {good }} \neq \emptyset\right\} .
\end{aligned}
$$


The lower and upper approximations of class $C l_{\text {good }}$ are the basis to induce "if ..., then ..." decision rules. These rules describe the conditions of assignment of the portfolios to class $C l_{\text {good }}$ (for an algorithm to induce decision rules, see $[8,30])$ :

- certain $\mathrm{D}_{\geq-}$decision rules, defining minimal performances to be attained on selected criteria from $F$ in order to assign with certainty portfolios $\mathbf{x}$ to class $C l_{\text {good }}$ : if $F_{i_{1}}(\mathbf{x}) \geq r_{i_{1}}$ and $\ldots$ and $F_{i_{q}}(\mathbf{x}) \geq r_{i_{q}}$, then certainly $\mathbf{x} \in C l_{\text {good }}$, where $r_{i_{1}}, \ldots, r_{i_{q}} \in \mathbb{R} ; F_{i_{1}}, \ldots, F_{i_{q}} \subseteq F$;

these decision rules are induced from $\underline{F}\left(C l_{\text {good }}\right)$, i.e., taking as positive examples to be matched the portfolios from $\underline{F}\left(C l_{\text {good }}\right)$;

- possible $\mathrm{D}_{\geq}$-decision rules, defining minimal performances to be attained on selected criteria from $F$ in order to assign with some possible doubt portfolios $\mathbf{x}$ to class $C l_{\text {good }}$ :

if $F_{i_{1}}(\mathbf{x}) \geq s_{i_{1}}$ and $\ldots$ and $F_{i_{p}}(\mathbf{x}) \geq s_{i_{q}}$, then possibly $\mathbf{x} \in C l_{\text {good }}$, where $s_{i_{1}}, \ldots, s_{i_{p}} \in \mathbb{R} ; F_{i_{1}}, \ldots, F_{i_{p}} \subseteq F$;

these decision rules are induced from $\bar{F}\left(C l_{\text {good }}\right)$, i.e., taking as positive examples to be matched the portfolios from $\bar{F}\left(C l_{\text {good }}\right)$.

A set of certain decision rules covering all consistent portfolios from class $C l_{\text {good }}$ represent aspirations of the DM expressed through the assignment examples. As such, it is a local preference model of the DM.

Although one has proposed different algorithms for induction of decision rules based on DRSA (see, e.g., [68], [70], [30], and [35]), for the portfolio decision problems we are considering, we suggest to adopt an algorithm that supplies an exhaustive description, i.e., the whole set of decision rules for the assignments given by the DM. If the number of decision rules is large, then one can select the "most interesting" rules to be showed to the DM using some filter referring to the number of portfolios covered, and ensuring for each portfolio assigned to the class $C l_{\text {good }}$ the presence of at least one rule describing it.

Indeed, the main problems of the exhaustive description strategy are the complexity of the induction algorithms, that are exponential in the number of criteria, and the potentially large number of rules [34]. However, for the application to portfolio decision problems, the computation complexity cannot be large, because the set of criteria cannot be too numerous, since, on the contrary, the DM would have some difficulty to handle the information.

Let us conclude this section with a discussion about handling "inconsistent" preference information supplied by the DM during the decision process. In fact, in terms of DRSA, the only inconsistent information is given in the case when portfolio $x_{j}$ is dominating portfolio $x_{k}$ and, nevertheless, the DM assigns $x_{j}$ to class $C l_{\text {others }}$ and $x_{k}$ to class $C l_{\text {good }}$. In this case, the inconsistent information is not removed and the $\mathrm{DM}$ is not requested to revise her/his preferences in order to restore consistency. Indeed, the inconsistent classification of $x_{j}$ and $x_{k}$ implies that both of them are included in $\bar{F}\left(C l_{\text {good }}\right)$ and none of them is included in $\underline{F}\left(C l_{\text {good }}\right)$. Thus, the inconsistency of the assignment of $x_{j}$ and $x_{k}$ is taken into consideration because they would not contribute to the induction of certain $\mathrm{D}_{\geq-}$decision rules which are based on $\underline{F}\left(C l_{\text {good }}\right)$, but they would contribute to the induction of possible $\mathrm{D}_{\geq-}$-decision rules that are based on $\bar{F}\left(C l_{\text {good }}\right)$. However, 
in the proposed procedure, the presence of inconsistent preferences has a limited impact because we present only non-dominated portfolios to the DM. Thus, in the set of portfolios that the DM is asked to evaluate, there is no pair $x_{j}$ and $x_{k}$ such that $x_{j}$ dominates $x_{k}$. Consequently, the DM cannot provide inconsistent preference information.

\subsection{Interactive Multi-objective Optimization guided by rules generated with DRSA (IMO-DRSA)}

Interactive Multi-objective Optimization (IMO) methods (for a survey, see [57]) alternate computation and dialog phases. In our cases, in the computation phase, a small set of weakly non-dominated portfolios with respect to the considered criteria (up to 20) is generated. In the dialog phase, the DM is invited to indicate some prospective portfolios, making a binary partition of this set into classes "good" and "others". The rules induced from such indirect preference information are used to define the constraints that reduce the solution space in the next calculation phase, thus, focusing the search on the region of feasible portfolios being more appealing to the DM.

Let $\mathbf{X}$ represent a set of portfolios in the current iteration, $\mathbf{x}$ be an individual portfolio, and $F_{i} \in F$ : $X \rightarrow \mathbb{R}, i=1, \ldots, m$ - the considered objective functions (criteria) to be maximized. The algorithm of IMO-DRSA is composed of the following steps [26]:

Step 1. Generate a representative sample $\mathbf{X}$ of feasible non-dominated portfolios.

Step 2. Present sample $\mathbf{X}$ to the DM.

Step 3. If the DM is satisfied with one portfolio $\mathbf{x} \in \mathbf{X}$, it is deemed as the compromise portfolio and the procedure stops. Otherwise, continue.

Step 4. Ask the DM to indicate in sample $\mathbf{X}$ a subset of "good" portfolios. The remaining portfolios are assumed to be classified as "others".

Step 5. Using DRSA, induce a set of decision rules covering all portfolios belonging to the lower approximation of class "good", i.e., to $\underline{F}\left(C l_{\text {good }}\right)$. The decision rules will have the following syntax: "if $F_{i_{1}}(\mathbf{x}) \geq \alpha_{i_{1}}$ and $\ldots$ and $F_{i_{q}}(\mathbf{x}) \geq \alpha_{i_{q}}$, then portfolio $\mathbf{x}$ is certainly good", $\left\{F_{i_{1}}, \ldots, F_{i_{q}}\right\} \subseteq F$. In case some objective function is to be minimized, the condition concerning this objective in the decision rule would have the form $F_{i}(\mathbf{x}) \leq \alpha_{i}$.

Step 6. Present the obtained set of rules to the DM.

Step 7. Ask the DM to select the decision rule(s) being the most representative of her/his preferences.

Step 8. Adjoin the constraints $F_{i_{1}}(\mathbf{x}) \geq \alpha_{i_{1}}, \ldots, F_{i_{q}}(\mathbf{x}) \geq \alpha_{i_{q}}$ coming from the rule(s) selected in Step 7 to the set of constraints of the multi-objective optimization problem at hand, in order to define the region of the most prospective portfolios for the DM's preferences. 
Step 9. Go back to Step 1.

Let us observe that the DM can come back to some previous iteration of the procedure, and to restart from there in order to explore different parts of the solution space. This is particularly appealing in a learning oriented perspective in which the interactive procedure is intended to permit the DM to acquire knowledge about both her/his preferences and possible trade-offs between objectives in the set of feasible portfolios.

\section{Illustrative example}

We illustrate the proposed method with the following hypothetical decision problem. Let us suppose that a funding council is expected to select a portfolio of research projects to be realized (for a review of applications in this field, see [60]). In particular, we consider an example involving 20 projects evaluated in terms of the following five criteria [79]:

- scientific excellence of the project $\left(g_{1}\right)$;

- relevance of the proposal to the priority areas of the council $\left(g_{2}\right)$;

- potential contribution towards advancing the research field $\left(g_{3}\right)$;

- experience of the researcher team submitting the project $\left(g_{4}\right)$;

- impact of the project (i.e., the contribution that it makes to the society and the economy) $\left(g_{5}\right)$.

For the sake of the simplicity, we give an evaluation of each project on each criterion on a scale $[0,100]$ (see Table 3). Note, however, that our model can work with any type of quantitative evaluations. Moreover, each project has an associated cost (in thousand Euro) which is also reported in Table 3.

For each criterion, the company's management has defined three satisfaction levels (see Table 4). We suppose the same levels are considered for all criteria. The available budget is equal to 340 .

The first sample of weakly non-dominated portfolios shown in Table 5 was obtained by solving 15 singleobjective 0-1 optimization problems relative to the maximization of $F_{t, i}$, for $t=1,2,3$, and $i=1,2,3,4,5$, as explained in Section 3. For example, when $F_{1,1}$ is accounted for, the single objective linear problem has been formulated as follows:

$$
\max \sum_{a_{j} \in A} h_{1, j, 1} x_{j}, \text { subject to } \sum_{a_{j} \in A} c_{j} x_{j} \leq C .
$$

Portfolio $P 1$, constructed by solving the above problem is composed of seven projects: $a_{1}, a_{5}, a_{6}, a_{7}, a_{8}, a_{11}, a_{14}$. Seven projects have an evaluation for the first criterion not less than $l_{1,1}=15$. Portfolio $P 2$ has been obtained by maximizing $F_{1,2}$ (see Table 5 ), etc. These problems have been solved with ILOG CPLEX V12.1 using Intel Core i7-4700MQ CPU, $2.40 \mathrm{GHz} \times 4$, with $8 \mathrm{~GB}$ of RAM memory. For each portfolio, we report the $F_{t, i}$ values. 
Tab. 3: Evaluations on five criteria and associated costs for twenty projects considered in the illustrative example.

\begin{tabular}{|c||c|c|c|c|c|c|}
\hline Projects & $g_{1}$ & $g_{2}$ & $g_{3}$ & $g_{4}$ & $g_{5}$ & Cost \\
\hline \hline$a_{1}$ & 18 & 41 & 42 & 15 & 18 & 37 \\
$a_{2}$ & 24 & 46 & 38 & 15 & 16 & 139 \\
$a_{3}$ & 43 & 41 & 54 & 55 & 55 & 123 \\
$a_{4}$ & 36 & 41 & 49 & 31 & 38 & 119 \\
$a_{5}$ & 19 & 48 & 60 & 10 & 48 & 46 \\
$a_{6}$ & 28 & 60 & 47 & 11 & 41 & 49 \\
$a_{7}$ & 60 & 49 & 54 & 51 & 16 & 60 \\
$a_{8}$ & 43 & 26 & 22 & 53 & 12 & 54 \\
$a_{9}$ & 14 & 12 & 55 & 33 & 25 & 119 \\
$a_{10}$ & 59 & 31 & 57 & 51 & 38 & 111 \\
$a_{11}$ & 56 & 13 & 15 & 59 & 60 & 47 \\
$a_{12}$ & 60 & 55 & 21 & 13 & 33 & 119 \\
$a_{13}$ & 36 & 34 & 26 & 29 & 34 & 63 \\
$a_{14}$ & 34 & 13 & 35 & 16 & 52 & 45 \\
$a_{15}$ & 27 & 47 & 34 & 14 & 21 & 130 \\
$a_{16}$ & 51 & 43 & 12 & 25 & 28 & 81 \\
$a_{17}$ & 34 & 14 & 29 & 24 & 36 & 120 \\
$a_{18}$ & 24 & 17 & 21 & 47 & 22 & 84 \\
$a_{19}$ & 12 & 13 & 21 & 44 & 16 & 120 \\
$a_{20}$ & 55 & 49 & 42 & 29 & 12 & 100 \\
\hline
\end{tabular}

Tab. 4: Satisfaction levels for five criteria considered in the illustrative example.

\begin{tabular}{|l||c|c|c|c|c|}
\hline Satisfaction levels & $g_{1}$ & $g_{2}$ & $g_{3}$ & $g_{4}$ & $g_{5}$ \\
\hline \hline$l_{1}:$ Satisfactory & 15 & 15 & 15 & 15 & 15 \\
$l_{2}:$ Very satisfactory & 30 & 30 & 30 & 30 & 30 \\
$l_{3}:$ Extremely satisfactory & 40 & 40 & 40 & 40 & 40 \\
\hline
\end{tabular}


Tab. 5: A set of non-dominated portfolios presented to the DM in the first iteration.

\begin{tabular}{|c||c|c|c|c|c|c|c|c|c|c|c|c|c|c|c|c|}
\hline Portfolio & $F_{1,1}$ & $F_{1,2}$ & $F_{1,3}$ & $F_{1,4}$ & $F_{1,5}$ & $F_{2,1}$ & $F_{2,2}$ & $F_{2,3}$ & $F_{2,4}$ & $F_{2,5}$ & $F_{3,1}$ & $F_{3,2}$ & $F_{3,3}$ & $F_{3,4}$ & $F_{3,5}$ & Class \\
\hline \hline$P 1$ & 7 & 5 & 7 & 5 & 6 & 4 & 4 & 5 & 3 & 4 & 3 & 4 & 4 & 3 & 4 & good \\
$P 2$ & 6 & 6 & 5 & 4 & 5 & 3 & 5 & 4 & 2 & 2 & 3 & 5 & 4 & 2 & 2 & good \\
$P 3$ & 7 & 5 & 7 & 5 & 6 & 4 & 4 & 5 & 3 & 4 & 3 & 4 & 4 & 3 & 4 & good \\
$P 4$ & 6 & 4 & 5 & 6 & 5 & 5 & 3 & 3 & 3 & 2 & 4 & 3 & 2 & 3 & 2 & $*$ \\
$P 5$ & 6 & 4 & 6 & 4 & 6 & 3 & 4 & 5 & 2 & 5 & 2 & 3 & 4 & 2 & 4 & $*$ \\
$P 6$ & 6 & 4 & 5 & 5 & 5 & 5 & 3 & 3 & 3 & 3 & 4 & 3 & 2 & 3 & 3 & $*$ \\
$P 7$ & 6 & 4 & 5 & 5 & 5 & 5 & 3 & 3 & 3 & 3 & 4 & 3 & 2 & 3 & 3 & $*$ \\
$P 8$ & 6 & 5 & 6 & 4 & 5 & 3 & 5 & 6 & 1 & 3 & 2 & 5 & 5 & 1 & 3 & good \\
$P 9$ & 6 & 5 & 6 & 4 & 5 & 3 & 3 & 3 & 4 & 3 & 3 & 3 & 3 & 4 & 3 & $*$ \\
$P 10$ & 6 & 4 & 6 & 4 & 6 & 4 & 4 & 4 & 2 & 5 & 2 & 3 & 3 & 2 & 4 & $*$ \\
$P 11$ & 6 & 4 & 5 & 5 & 5 & 5 & 3 & 3 & 3 & 3 & 4 & 3 & 2 & 3 & 3 & $*$ \\
$P 12$ & 6 & 5 & 6 & 4 & 5 & 3 & 5 & 5 & 2 & 3 & 3 & 5 & 5 & 2 & 3 & good \\
$P 13$ & 6 & 5 & 6 & 4 & 5 & 3 & 5 & 5 & 2 & 3 & 3 & 5 & 5 & 2 & 3 & good \\
$P 14$ & 5 & 4 & 5 & 4 & 4 & 4 & 3 & 3 & 4 & 3 & 4 & 3 & 3 & 4 & 3 & $*$ \\
$P 15$ & 5 & 3 & 5 & 3 & 5 & 3 & 3 & 4 & 2 & 5 & 2 & 3 & 3 & 2 & 5 & $*$ \\
\hline
\end{tabular}

Note that the following three pairs of portfolios are the same: $(P 1, P 3),(P 6, P 7)$, and $(P 12, P 13)$. The non-dominated portfolios are presented to the DM as histograms (see Figure 1) which display the numbers of projects with an evaluation not less than the satisfaction threshold $l_{t, i}$ for each $g_{i} \in G$ and for each $l_{t, i} \in L_{i}$. Such graphical representation is used because it supports the intuition of the DM, making the method applicable even when dealing with higher level managers often inhibited by adopting more sophisticated and complex decision support models [23]. Let us suppose that the DM evaluates the 15 portfolios and indicates those which are relatively "good", as shown in the last column of Table 5.

To illustrate how the DM can reason on the comprehensive quality of the projects, let us provide the following simple example. The first portfolio $(P 1)$ has been obtained by optimizing the number of projects that have an evaluation at least equal to the first satisfaction level for $g_{1}$. As a result, it contains seven projects that are at least satisfactory in terms of the scientific excellence of the project. Among them, there are four projects that are very satisfactory and three projects that are extremely satisfactory on $g_{1}$. The second portfolio $(P 2)$ has been obtained by optimizing the number of projects that have an evaluation at least equal to the first satisfaction level for $g_{2}$. Thus, it contains more projects that are favorable in terms of the relevance of the proposal for the council $\left(g_{2}\right)$ rather than in terms of their scientific excellence $\left(g_{1}\right)$. Taking these arguments into account, the DM is supposed to indicate which portfolios better correspond to her/his preferences. Obviously, a different decision could be made by the DM who is a member of the council or the DM being an external reviewer.

Using DRSA to preference information presented in Table 5, one obtains 41 decision rules matching the portfolios evaluated as "good". The most interesting decision rules, which are supported by a relatively high number of "good" portfolios, are the following (in the parentheses we provide the identifiers of portfolios 

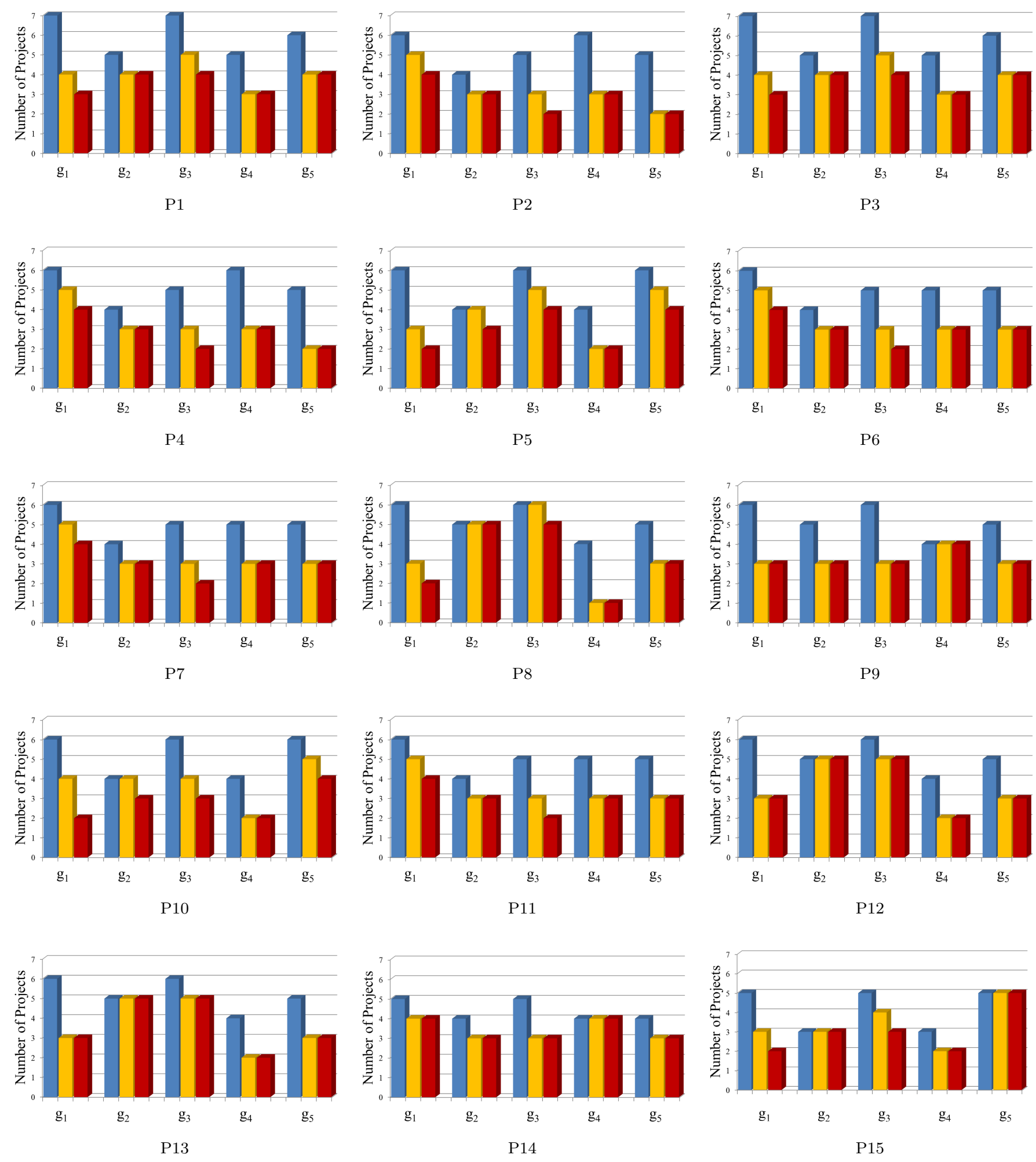

At Least Satisfactory $\quad$ At Least Very Satisfactory $\quad$ Extremely Satisfactory

Fig. 1: Histograms representing the set of non-dominated portfolios in the first iteration. 
supporting the corresponding rule):

Rule 1.1: if $F_{1,2}(\mathbf{x}) \geq 5$ and $F_{2,3}(\mathbf{x}) \geq 5$, then portfolio $\mathbf{x}$ is "good", $(P 1, P 3, P 8, P 12, P 13)$

(if there are at least 5 projects satisfactory or better with respect to the relevance of the priority areas of the council and at least five projects very satisfactory or better with respect to potential contributions towards advancing the research field, then the portfolio is good);

Rule 1.2: if $F_{2,2}(\mathbf{x}) \geq 4$ and $F_{3,1}(\mathbf{x}) \geq 3$, then portfolio $\mathbf{x}$ is "good",

$(P 1, P 2, P 3, P 12, P 13)$

(if there are at least 4 projects very satisfactory or better with respect to the relevance of the priority areas of the council and at least 3 projects extremely satisfactory with respect to the scientific excellence, then the portfolio is good);

Rule 1.3: if $F_{1,2}(\mathbf{x}) \geq 5$ and $F_{2,2}(\mathbf{x}) \geq 4$, then portfolio $\mathbf{x}$ is "good",

$(P 1, P 2, P 3, P 8, P 12, P 13)$

(if there are at least 5 projects satisfactory or better with respect to the relevance of the priority areas of the council and of these at least 4 projects are very satisfactory or better, then the portfolio is good);

Rule 1.4: if $F_{2,3}(\mathbf{x}) \geq 4$ and $F_{3,1}(\mathbf{x}) \geq 3$, then portfolio $\mathbf{x}$ is "good",

$(P 1, P 2, P 3, P 12, P 13)$

(if there are at least 4 projects very satisfactory or better with respect to the potential contribution towards advancing the research field and at least 3 projects extremely satisfactory with respect to the scientific excellence, then the portfolio is good);

Rule 1.5: if $F_{3,2}(\mathbf{x}) \geq 4$, then portfolio $\mathbf{x}$ is "good",

$(P 1, P 2, P 3, P 8, P 12, P 13)$

(if there are at least 4 projects extremely satisfactory with respect to the relevance of the priority areas of the council, then the portfolio is good);

Rule 1.6: if $F_{1,2}(\mathbf{x}) \geq 5$ and $F_{3,3}(\mathbf{x}) \geq 4$, then portfolio $\mathbf{x}$ is "good",

$(P 1, P 2, P 3, P 8, P 12, P 13)$

(if there are at least 5 projects satisfactory or better with respect to the relevance of the priority areas of the council and at least 4 projects extremely satisfactory with respect to the potential contributions towards advancing the research field, then the portfolio is good);

Rule 1.7: if $F_{3,1}(\mathbf{x}) \geq 3$ and $F_{3,3}(\mathbf{x}) \geq 4$, then portfolio $\mathbf{x}$ is "good",

$(P 1, P 2, P 3, P 12, P 13)$.

(if there are at least 3 projects extremely satisfactory with respect to the scientific excellence and at least 4 projects extremely satisfactory with respect to the potential contributions towards advancing the research field, then the portfolio is good).

The DM selected Rule 1.3 as the most representative for her/his current aspirations, and thus, the following constraints were added to the original optimization problem: 
Tab. 6: A set of non-dominated portfolios presented to the DM in the second iteration.

\begin{tabular}{|c||c|c|c|c|c|c|c|c|c|c|c|c|c|c|c|c|}
\hline Portfolio & $F_{1,1}$ & $F_{1,2}$ & $F_{1,3}$ & $F_{1,4}$ & $F_{1,5}$ & $F_{2,1}$ & $F_{2,2}$ & $F_{2,3}$ & $F_{2,4}$ & $F_{2,5}$ & $F_{3,1}$ & $F_{3,2}$ & $F_{3,3}$ & $F_{3,4}$ & $F_{3,5}$ & Class \\
\hline \hline$P 1^{\prime}$ & 7 & 5 & 7 & 5 & 6 & 4 & 4 & 5 & 3 & 4 & 3 & 4 & 4 & 3 & 4 & $*$ \\
$P 2^{\prime}$ & 6 & 6 & 6 & 4 & 5 & 3 & 5 & 4 & 2 & 3 & 2 & 4 & 4 & 2 & 2 & $*$ \\
$P 3^{\prime}$ & 7 & 5 & 7 & 5 & 6 & 4 & 4 & 5 & 3 & 4 & 3 & 4 & 4 & 3 & 4 & $*$ \\
$P 4^{\prime}$ & 6 & 5 & 5 & 6 & 5 & 5 & 4 & 3 & 2 & 2 & 3 & 3 & 2 & 2 & 1 & $*$ \\
$P 5^{\prime}$ & 6 & 5 & 6 & 4 & 6 & 3 & 5 & 4 & 2 & 4 & 2 & 4 & 4 & 2 & 3 & $*$ \\
$P 6^{\prime}$ & 7 & 5 & 7 & 5 & 6 & 4 & 4 & 5 & 3 & 4 & 3 & 4 & 4 & 3 & 4 & $*$ \\
$P 7^{\prime}$ & 6 & 6 & 5 & 4 & 6 & 3 & 6 & 4 & 1 & 3 & 2 & 5 & 4 & 1 & 2 & good \\
$P 8^{\prime}$ & 6 & 5 & 6 & 4 & 5 & 3 & 5 & 6 & 1 & 3 & 2 & 5 & 5 & 1 & 3 & good \\
$P 9^{\prime}$ & 7 & 5 & 7 & 5 & 6 & 4 & 4 & 5 & 3 & 4 & 3 & 4 & 4 & 3 & 4 & $*$ \\
$P 10^{\prime}$ & 6 & 5 & 6 & 4 & 6 & 3 & 5 & 4 & 2 & 4 & 2 & 4 & 4 & 2 & 3 & $*$ \\
$P 11^{\prime}$ & 6 & 5 & 5 & 5 & 5 & 4 & 4 & 3 & 3 & 2 & 4 & 4 & 3 & 3 & 2 & $*$ \\
$P 12^{\prime}$ & 6 & 5 & 6 & 4 & 5 & 3 & 5 & 5 & 2 & 3 & 3 & 5 & 5 & 2 & 3 & good \\
$P 13^{\prime}$ & 6 & 5 & 6 & 4 & 5 & 3 & 5 & 5 & 2 & 3 & 3 & 5 & 5 & 2 & 3 & good \\
$P 14^{\prime}$ & 7 & 5 & 7 & 5 & 6 & 4 & 4 & 5 & 3 & 4 & 3 & 4 & 4 & 3 & 4 & $*$ \\
$P 15^{\prime}$ & 7 & 5 & 7 & 5 & 6 & 4 & 4 & 5 & 3 & 4 & 3 & 4 & 4 & 3 & 4 & $*$ \\
\hline
\end{tabular}

$$
F_{1,2}(\mathbf{x}) \geq 5 \text { and } F_{2,2}(\mathbf{x}) \geq 4
$$

The DM seems to prefer a portfolio composed of the maximum number of projects being satisfactory or very satisfactory for the second criterion related to the relevance of the proposal for the council.

Then, the second sample of weakly non-dominated portfolios shown in Table 6 was generated and presented to the DM (to save space, we do not include the histograms corresponding to the considered portfolios). Note that portfolios $P 1^{\prime}, P 3^{\prime}, P 6^{\prime}, P 9^{\prime}, P 14^{\prime}$ and $P 15^{\prime}$ are the same, so as portfolios $P 12^{\prime}$ and $P 13^{\prime}$. Upon reflection, the DM indicated the portfolios considered as relatively "good" in the second sample. This information is displayed in the last column of Table 6 .

Applying DRSA to preference information contained in Table 6, one obtains the following 7 decision rules describing portfolios deemed as "good" (to save space, in what follows, we do not provide the formulation of rules in the natural language):

Rule 2.1: if $F_{1,2}(\mathbf{x}) \geq 6$ and $F_{1,5}(\mathbf{x}) \geq 6$, then portfolio $\mathbf{x}$ is "good",

Rule 2.2: if $F_{2,2}(\mathbf{x}) \geq 6$, then portfolio $\mathbf{x}$ is "good",

Rule 2.3: if $F_{2,3}(\mathbf{x}) \geq 6$, then portfolio $\mathbf{x}$ is "good",

Rule 2.4: if $F_{2,2}(\mathbf{x}) \geq 5$ and $F_{2,3}(\mathbf{x}) \geq 5$, then portfolio $\mathbf{x}$ is "good",

$\left(P 8^{\prime}, P 12^{\prime}, P 13^{\prime}\right)$

Rule 2.5: if $F_{2,2}(\mathbf{x}) \geq 5$ and $F_{3,1}(\mathbf{x}) \geq 3$, then portfolio $\mathbf{x}$ is "good",

$\left(P 12^{\prime}, P 13^{\prime}\right)$

Rule 2.6: if $F_{3,2}(\mathbf{x}) \geq 5$, then portfolio $\mathbf{x}$ is "good",

$\left(P 7^{\prime}, P 8^{\prime}, P 12^{\prime}, P 13^{\prime}\right)$ 
Tab. 7: A set of non-dominated portfolios presented to the DM in the third iteration.

\begin{tabular}{|c||c|c|c|c|c|c|c|c|c|c|c|c|c|c|c|c|}
\hline Portfolio & $F_{1,1}$ & $F_{1,2}$ & $F_{1,3}$ & $F_{1,4}$ & $F_{1,5}$ & $F_{2,1}$ & $F_{2,2}$ & $F_{2,3}$ & $F_{2,4}$ & $F_{2,5}$ & $F_{3,1}$ & $F_{3,2}$ & $F_{3,3}$ & $F_{3,4}$ & $F_{3,5}$ & Class \\
\hline \hline$P 1^{\prime \prime}$ & 6 & 5 & 5 & 4 & 6 & 3 & 5 & 5 & 1 & 3 & 2 & 5 & 4 & 1 & 3 & $*$ \\
$P 2^{\prime \prime}$ & 6 & 6 & 5 & 4 & 6 & 3 & 6 & 4 & 1 & 3 & 2 & 5 & 4 & 1 & 2 & good \\
$P 3^{\prime \prime}$ & 6 & 5 & 6 & 4 & 5 & 3 & 5 & 6 & 1 & 3 & 2 & 5 & 5 & 1 & 3 & $*$ \\
$P 4^{\prime \prime}$ & 6 & 5 & 5 & 4 & 6 & 3 & 5 & 5 & 1 & 3 & 2 & 5 & 4 & 1 & 3 & $*$ \\
$P 5^{\prime \prime}$ & 6 & 6 & 5 & 4 & 6 & 3 & 6 & 4 & 1 & 3 & 2 & 5 & 4 & 1 & 2 & good \\
$P 6^{\prime \prime}$ & 6 & 5 & 6 & 4 & 5 & 3 & 5 & 5 & 2 & 3 & 3 & 5 & 5 & 2 & 3 & $*$ \\
$P 7^{\prime \prime}$ & 6 & 6 & 5 & 4 & 6 & 3 & 6 & 4 & 1 & 3 & 2 & 5 & 4 & 1 & 2 & good \\
$P 8^{\prime \prime}$ & 6 & 5 & 6 & 4 & 5 & 3 & 5 & 6 & 1 & 3 & 2 & 5 & 5 & 1 & 3 & $*$ \\
$P 9^{\prime \prime}$ & 6 & 5 & 6 & 4 & 5 & 3 & 5 & 5 & 2 & 3 & 3 & 5 & 5 & 2 & 3 & $*$ \\
$P 10^{\prime \prime}$ & 6 & 5 & 6 & 4 & 5 & 3 & 5 & 5 & 2 & 3 & 3 & 5 & 5 & 2 & 3 & $*$ \\
$P 11^{\prime \prime}$ & 6 & 6 & 5 & 4 & 5 & 3 & 5 & 4 & 2 & 2 & 3 & 5 & 4 & 2 & 2 & $*$ \\
$P 12^{\prime \prime}$ & 6 & 5 & 6 & 4 & 5 & 3 & 5 & 5 & 2 & 3 & 3 & 5 & 5 & 2 & 3 & $*$ \\
$P 13^{\prime \prime}$ & 6 & 5 & 6 & 4 & 5 & 3 & 5 & 5 & 2 & 3 & 3 & 5 & 5 & 2 & 3 & $*$ \\
$P 14^{\prime \prime}$ & 6 & 5 & 5 & 4 & 6 & 3 & 5 & 4 & 2 & 3 & 3 & 5 & 4 & 2 & 3 & $*$ \\
$P 15^{\prime \prime}$ & 6 & 5 & 5 & 4 & 6 & 3 & 5 & 5 & 1 & 3 & 2 & 5 & 4 & 1 & 3 & $*$ \\
\hline
\end{tabular}

Rule 2.7: if $F_{3,3}(\mathbf{x}) \geq 5$, then portfolio $\mathbf{x}$ is "good".

$\left(P 8^{\prime}, P 12^{\prime}, P 13^{\prime}\right)$

In the second dialog phase, the DM selected Rule 2.6 as the most representative of her/his current aspirations. Thus, the DM wants to maximize the number of projects that are extremely satisfactory for the second criterion. To allow this, the following constraint was added to the optimization problem from the second computation phase:

$$
F_{3,2}(\mathbf{x}) \geq 5
$$

Next, the third sample of non-dominated portfolios shown in Table 7 was generated and presented to the DM. The groups of equivalent portfolios include: (P1" $\left.P 4^{\prime \prime}, P 15^{\prime \prime}\right),\left(P 2^{\prime \prime}, P 5^{\prime \prime}, P 7^{\prime \prime}\right),\left(P 3^{\prime \prime}, P 6^{\prime \prime}\right.$, $\left.P 8^{\prime \prime}\right)$, and $\left(P 9^{\prime \prime}, P 10^{\prime \prime}, P 12^{\prime \prime}, P 13^{\prime \prime}\right)$. As before, the DM evaluated some portfolios as relatively "good" (this information is displayed in the last column of Table 7). Applying DRSA to preference information contained in Table 7, one obtains the following 3 decision rules matching all "good" portfolios:

Rule 3.1: if $F_{1,2}(\mathbf{x}) \geq 6$ and $F_{1,5}(\mathbf{x}) \geq 6$, then portfolio $\mathbf{x}$ is "good",

Rule 3.2: if $F_{2,2}(\mathbf{x}) \geq 6$, then portfolio $\mathbf{x}$ is "good",

Rule 3.3: if $F_{1,2}(\mathbf{x}) \geq 6$ and $F_{2,5}(\mathbf{x}) \geq 3$, then portfolio $\mathbf{x}$ is "good".

$\left(P 2^{\prime}, P 5^{\prime}, P 7^{\prime}\right)$

For example, Rule 3.3 highlights how the DM prefers to have at least 6 projects that are satisfactory or better in terms of the impact of the project $\left(g_{5}\right)$.

At this stage, it has been noticed that selection of any of the three rules for inclusion of its elementary conditions in the constraints of the optimization problem from the third computation phase leads to a set 
Tab. 8: A set of non-dominated portfolios presented to the DM in the fourth iteration.

\begin{tabular}{|c||c|c|c|c|c|c|c|c|c|c|c|c|c|c|c|c|}
\hline Portfolio & $F_{1,1}$ & $F_{1,2}$ & $F_{1,3}$ & $F_{1,4}$ & $F_{1,5}$ & $F_{2,1}$ & $F_{2,2}$ & $F_{2,3}$ & $F_{2,4}$ & $F_{2,5}$ & $F_{3,1}$ & $F_{3,2}$ & $F_{3,3}$ & $F_{3,4}$ & $F_{3,5}$ & Class \\
\hline \hline$P 1^{\prime \prime \prime}$ & 6 & 6 & 5 & 4 & 6 & 3 & 6 & 4 & 1 & 3 & 2 & 5 & 4 & 1 & 2 & good \\
$P 2^{\prime \prime \prime}$ & 6 & 6 & 5 & 4 & 6 & 3 & 6 & 4 & 1 & 3 & 2 & 5 & 4 & 1 & 2 & good \\
$P 3^{\prime \prime \prime}$ & 6 & 6 & 5 & 4 & 6 & 3 & 6 & 4 & 1 & 3 & 2 & 5 & 4 & 1 & 2 & good \\
$P 4^{\prime \prime \prime}$ & 6 & 6 & 5 & 4 & 6 & 3 & 6 & 4 & 1 & 3 & 2 & 5 & 4 & 1 & 2 & good \\
$P 5^{\prime \prime \prime}$ & 6 & 6 & 5 & 4 & 6 & 3 & 6 & 4 & 1 & 3 & 2 & 5 & 4 & 1 & 2 & good \\
$P 6^{\prime \prime \prime}$ & 6 & 6 & 5 & 4 & 6 & 3 & 6 & 4 & 1 & 3 & 2 & 5 & 4 & 1 & 2 & good \\
$P 7^{\prime \prime \prime}$ & 6 & 6 & 5 & 4 & 6 & 3 & 6 & 4 & 1 & 3 & 2 & 5 & 4 & 1 & 2 & good \\
$P 8^{\prime \prime \prime}$ & 6 & 6 & 5 & 4 & 6 & 3 & 6 & 4 & 1 & 3 & 2 & 5 & 4 & 1 & 2 & good \\
$P 9^{\prime \prime \prime}$ & 6 & 6 & 5 & 4 & 6 & 3 & 6 & 4 & 1 & 3 & 2 & 5 & 4 & 1 & 2 & good \\
$P 10^{\prime \prime \prime}$ & 6 & 6 & 5 & 4 & 6 & 3 & 6 & 4 & 1 & 3 & 2 & 5 & 4 & 1 & 2 & good \\
$P 11^{\prime \prime \prime}$ & 6 & 6 & 5 & 4 & 6 & 3 & 6 & 4 & 1 & 3 & 2 & 5 & 4 & 1 & 2 & good \\
$P 12^{\prime \prime \prime}$ & 6 & 6 & 5 & 4 & 6 & 3 & 6 & 4 & 1 & 3 & 2 & 5 & 4 & 1 & 2 & good \\
$P 13^{\prime \prime \prime}$ & 6 & 6 & 5 & 4 & 6 & 3 & 6 & 4 & 1 & 3 & 2 & 5 & 4 & 1 & 2 & good \\
$P 14^{\prime \prime \prime}$ & 6 & 6 & 5 & 4 & 6 & 3 & 6 & 4 & 1 & 3 & 2 & 5 & 4 & 1 & 2 & good \\
$P 15^{\prime \prime \prime}$ & 6 & 6 & 5 & 4 & 6 & 3 & 6 & 4 & 1 & 3 & 2 & 5 & 4 & 1 & 2 & good \\
\hline
\end{tabular}

of the same portfolios shown in Table 8 and Figure 2, including projects: $a_{1}, a_{5}, a_{6}, a_{7}, a_{13}$, and $a_{16}$. The DM found this portfolio to be "good", which concluded the interactive procedure.

Let us remark that the finally chosen portfolio satisfies all the decision rules selected by the DM in each step of the interactive procedure. Thus, the conditions of these rules can be considered as arguments indicating an explanation of the final decision. In other words, in the considered example, supposing that in the last iteration the DM selected Rule 3.1 as the most representative one, the final decision can be justified with the following arguments: in the considered portfolio:

Argument 1) there are at least five projects that are at least satisfactory with respect to $g_{2}$

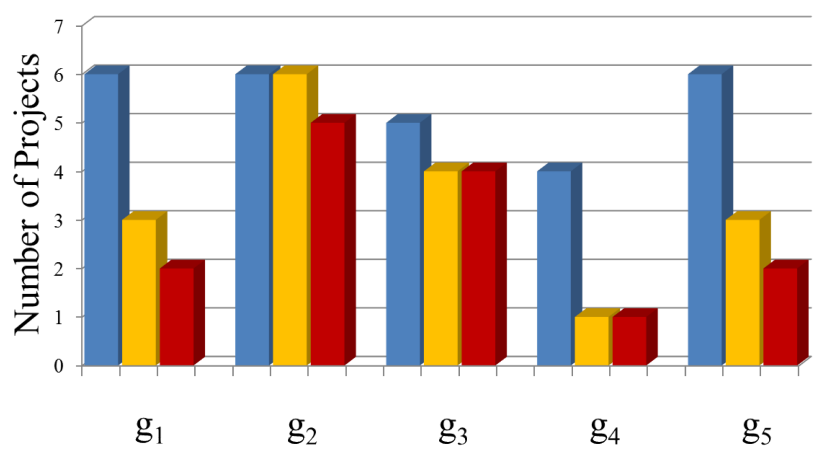

-At Least Satisfactory $\quad$ At Least Very Satisfactory $\quad$ Extremely Satisfactory

Fig. 2: Histogram representing the non-dominated portfolio in the fourth iteration. 
(i.e., $F_{1,2}(\mathbf{x}) \geq 5$, by Rule 1.3$)$,

Argument 2) there are at least four projects that are at least very satisfactory with respect to $g_{2}$ (i.e., $F_{2,2}(\mathbf{x}) \geq 4$, by Rule 1.3 ),

Argument 3 ) there are at least five projects that are at least extremely satisfactory with respect to $g_{2}$ (i.e., $F_{3,2}(\mathbf{x}) \geq 5$, by Rule 2.6 ),

Argument 4) there are at least six projects that are at least satisfactory with respect to $g_{2}$ (i.e., $F_{1,2}(\mathbf{x}) \geq 6$, by Rule 3.1$)$, and

Argument 5) there are at least six projects that are at least satisfactory with respect to $g_{5}$ (i.e., $F_{1,5}(\mathbf{x}) \geq 6$, by Rule 3.1$)$.

With Arguments 1) and 2) being implied by Argument 3), the justification of the final decision is based on Arguments 3),4), and 5). The DM was very keen on two particular criteria: the relevance of the projects for the council $\left(g_{2}\right)$ and the impact of the projects $\left(g_{5}\right)$. This is confirmed also by a redefinition of the rule concerning the relevance of the projects during the interaction phase, asking for an even more strict constraint on the number of portfolios extremely satisfactory on $g_{2}$.

Let us compare the solution obtained by the IMO-DRSA procedure with the solutions constructed by applying the linear value approach and the compromise programming procedure. As in the example in Section 3 , we adopted a value function with equal weights for all criteria $g_{i} \in G$, and a compromise programming based on the minimization of the maximum deviation from the ideal point. These two approaches led to the same portfolio whose histogram is presented in Figure 3. In fact, one can see that the portfolio obtained using IMO-DRSA is performing much better in terms of $g_{2}$, and more or less worse on the remaining criteria. Thus, since the process of selecting the portfolio by IMO-DRSA accounted for the critics of the DM on the reference portfolios showed in the successive iterations, we must conclude that for the DM, $g_{2}$ is the most important criterion.

For this reason, we have explored what would be the portfolios suggested by the other two approaches when increasing the weight $w_{2}$ associated with criterion $g_{2}$. More precisely, we considered $w_{2}$ equal to 1,2 , 3,4 , or 5 , while maintaining the weights of the remaining criteria equal to 1 . When maximizing the value function, for all considered settings we obtained the same portfolio $P 1$ presented in Figure 3 . We also used the compromise programming when multiplying $\Delta_{2}$ (i.e., the deviation of the performance on $g_{2}$ from the ideal point) by the weight $w_{2}$ equal to $1,2,3,4$, or 5 . For $w_{2}$ equal to 1,2 , and 3 , we obtained the same portfolio $P 1$ (see Figure 3), whereas with $w_{2}=4$ or 5 , the portfolio presented in Figure 4 was constructed. The latter portfolio has more advantageous performances on $g_{2}$ than the portfolio obtained by maximizing a value function though still it is not as favorable as the portfolio selected by the IMO-DRSA procedure. 


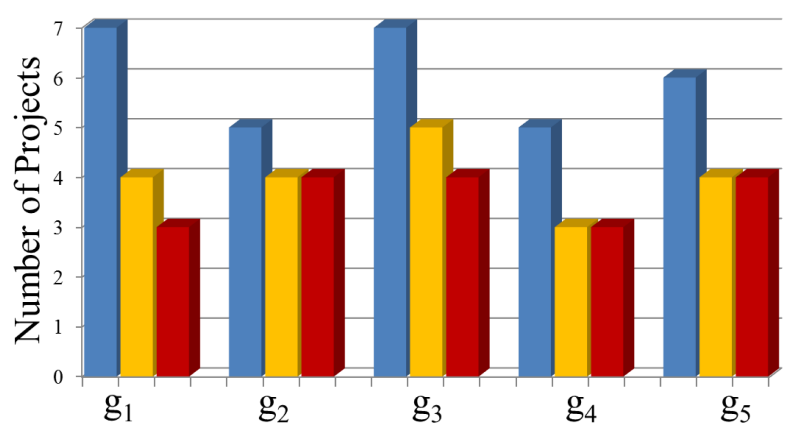

-At Least Satisfactory $\quad$ At Least Very Satisfactory $\quad$ Extremely Satisfactory

Fig. 3: Histogram representing the portfolio obtained by using a linear value approach and a compromise programming procedure with $w_{2}=1,2$, or 3 .

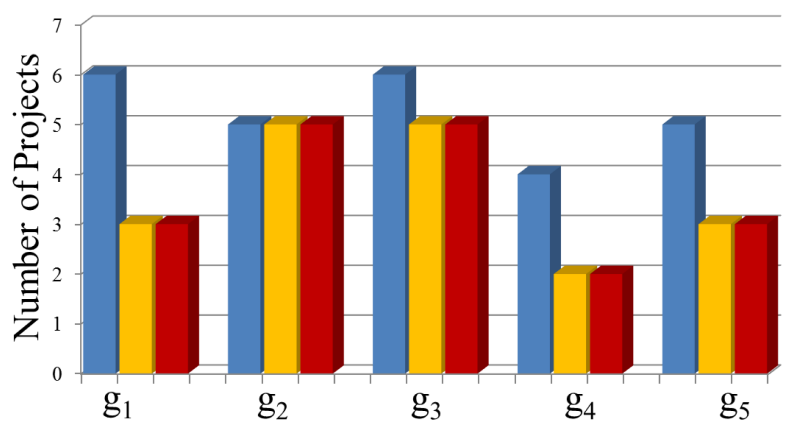

-At Least Satisfactory $\quad$ At Least Very Satisfactory $\quad$ Extremely Satisfactory

Fig. 4: Histogram representing the portfolio obtained using the compromise programming approach with $w_{2}=4$ or 5 . 


\section{Conclusion}

In this paper, we proposed a new methodology to deal with Portfolio Decision Problems. It is based on an interactive multi-objective optimization procedure, called IMO-DRSA (Interactive Multiobjective Optimization driven by Dominance-based Rough Set Approach), guided by decision rules. The latter ones are induced from the classification examples provided by the DM on a small sample of weakly non-dominated portfolios with respect to the objectives indicating the numbers of projects in a given portfolio that attain different pre-defined satisfaction levels on criteria used for evaluation of the projects. When analyzing a sample of the portfolios in the current iteration, the DM just indicates some prospective portfolios as "good" ones, opposing them against the "others". This classification data is structured using the Dominance-based Rough Set Approach and then decision rules matching the "good" portfolios are induced. These rules are expressed in an easily understandable "if ..., then ..." form. At each stage, the DM selects one decision rule that best represents her/his current aspirations. Then, elementary conditions of the selected rules are added to the constraints of the last multi-objective optimization problem, and a new sample of non-dominated portfolios is generated to be presented to the DM. The use of decision rules as a preference model has the advantage of favoring discussion and critical reflection of the DM in this interactive process.

The most relevant contribution of our approach is the formulation of the objective functions of the portfolio decision problem. We propose to maximize the number of projects in the portfolio which attain some pre-defined satisfaction levels such as "weakly satisfactory", "satisfactory", "very satisfactory", and "extremely satisfactory". This permits to avoid the risk of selecting portfolios composed of projects having good performances concentrated on one criterion or on a small set of criteria, as it might happen with the usual assessment of the portfolios in terms of the sum of marginal values assigned to component projects. We are convinced that the basic point in portfolio decision problems is that the number of projects attaining good levels of satisfaction should be well distributed among the considered criteria according to the nature of the problem and to the preferences of the decision maker. Moreover, strictly related to this point, is the specific method of interactive multi-objective optimization proposed. In fact, in this context, IMO-DRSA focuses the attention of the DM on the number of projects attaining pre-defined levels of satisfaction, with respect to the information supplied by the DM as well as with respect to the information given back to the DM. Indeed, let us remark that the DM is required to supply information in terms of classification of feasible portfolios described in terms of the number of projects attaining the pre-defined levels of satisfaction on the considered criteria. Moreover, the DM receives decision rules that explain her/his classification in terms of thresholds to be attained by the number of projects reaching the pre-defined levels of satisfaction on considered criteria. The practical use of the proposed methodology has been illustrated with a simple example, showing its potential for future real world applications.

We envisage a few directions of further research in Portfolio Decision Analysis. Our approach could be applied to specific portfolio problems by adding further constraints. For example, in a facility location problem [17], one additionally considers constraints related to the homogeneous cover of a location space, proximity to the users and to the related infrastructures, etc. Another further development consists in using 
the numbers of projects attaining some pre-defined satisfaction levels as arguments of value functions to be maximized, thus, extending our approach to preference models different than a set of decision rules. We recognize that the application of some effective heuristics could help solving big instances of portfolio problems. In this regard, we recommend the use of some Evolutionary Multi-objective Optimization algorithms guided by preference information supplied by the DM (see, e.g., [9, 10, 37, 38]).

To handle real-world problems more effectively, one could include aspects related to risk and uncertainty in the formulation of the problem. For example, having a probability distribution on a set of scenarios, one could consider as objectives to be maximized quantiles of the numbers of projects attaining some pre-defined levels of satisfaction (for dealing with risk and uncertainty using DRSA, see [27, 36], while for an application of IMO-DRSA to decision under risk and uncertainty, see [28]).

\section{Acknowledgment}

Salvatore Greco wishes to acknowledge funding by the "FIR of the University of Catania BCAEA3 New developments in Multiple Criteria Decision Aiding (MCDA) and their application to territorial competitiveness". The work of Miłosz Kadziński was supported by the Polish Ministry of Science and Higher Education under the Iuventus Plus program in 2016-2019 grant number IP2015 029674 - 0296/IP2/2016/74. The work of Roman Słowiński was supported by the Statutory Fund of the Poznań University of Technology, 09/91/DSPB/0602/503216.

\section{Bibliography}

\section{References}

[1] Almeida, J. A. D., Almeida, A. T. D., and Costa, A. P. (2014). Portfolio Selection of Information Systems Projects Using PROMETHEE V with C-Optimal Concept. Pesquisa Operacional, 34(2), 275-299.

[2] Álvarez-Miranda, E., Garcia-Gonzalo, J., Ulloa-Fierro, F., Weintraub, A., and Barreiro, S. (2017). A Multicriteria Optimization Model for Sustainable Forest Management under Climate Change Uncertainty: An application in Portugal. European Journal of Operational Research, https://doi.org/10.1016/j.ejor.2017.04.052

[3] Alves, M. J., and Climaco, J. (2007). A review of interactive methods for multiobjective integer and mixed-integer programming. European Journal of Operational Research, 180(1), 99-115.

[4] Archer, N. P., and Ghasemzadeh, F. (1999). An integrated framework for project portfolio selection. International Journal of Project Management, 17(4), 207-216.

[5] Argyris, N., Figueira, J. R., and Morton, A. (2011). Identifying preferred solutions to Multi-Objective 
Binary Optimisation problems, with an application to the Multi-Objective Knapsack Problem. Journal of Global Optimization, 49(2), 213-235.

[6] Badri, M. A., Davis, D., and Davis, D. (2001). A comprehensive 0-1 goal programming model for project selection. International Journal of Project Management, 19(4), 243-252.

[7] Bazgan, C., Hugot, H., and Vanderpooten, D. (2009). Solving efficiently the 0-1 multi-objective knapsack problem. Computers and Operations Research, 36(1), 260-279.

[8] Błaszczyński, J., Słowiński, R., Szeląg, J., (2011). Sequential covering rule induction algorithm for variable consistency rough set approaches. Information Sciences, 181(5), 987-1002.

[9] Branke, J., Corrente, S., Greco, S., Słowiński, R. and Zielniewicz, P. (2016). Using Choquet integral as preference model in interactive evolutionary multiobjective optimization. European Journal of Operational Research, 250(3), 884-901.

[10] Branke, J., Greco, S., Słowiński, R., Zielniewicz, P. (2015). Learning value functions in interactive evolutionary multiobjective optimization. Evolutionary Computation, IEEE Transactions on, 19(1), 88102.

[11] Brown, G. G., Dell, R. F., and Newman, A. M. (2004). Optimizing military capital planning. Interfaces, $34(6), 415-425$.

[12] Captivo, M. E., Climaco, J., Figueira, J., Martins, E., and Santos, J. L. (2003). Solving bicriteria 0-1 knapsack problems using a labeling algorithm. Computers and Operations Research, 30(12), 1865-1886.

[13] Cohon, J. L. (2013). Multiobjective programming and planning. Courier Corporation.

[14] da Silva, C. G., Climaco, J., and Figueira, J. (2006). A scatter search method for bi-criteria $\{0,1\}$ knapsack problems. European Journal of Operational Research, 169(2), 373-391.

[15] da Silva, C. G., Figueira, J., and Climaco, J. (2007). Integrating partial optimization with scatter search for solving bi-criteria $\{0,1\}$-knapsack problems. European Journal of Operational Research, 177(3), 16561677.

[16] da Silva, C. G., Meidanis, J., Moura, A. V., Souza, M. A., Viadanna, P., de Oliveira, M. R., de Oliveira, M.R., Lidianne, H. J, Costa Lima, A.R and de Barros, R. S. (2017). An improved visualization-based approach for project portfolio selection. Computers in Human Behavior, 73, 685696.

[17] Drezner, Z. (Ed.). (1995). Facility location: a survey of applications and methods. Springer.

[18] Drezner, T., Drezner, Z., and Salhi, S. (2006). A multi-objective heuristic approach for the casualty collection points location problem. Journal of the Operational Research Society, 57(6), 727-734. 
[19] Ehrgott, M., and Gandibleux, X. (2000). A survey and annotated bibliography of multiobjective combinatorial optimization. OR-Spektrum, 22(4), 425-460.

[20] Ehrgott, M., Gandibleux, X., and Przybylski, A. (2016). Exact Methods for Multi-Objective Combinatorial Optimisation. Chapter 19 [in]: S. Greco, J. Figueira, and M. Ehrgott (eds.), Multiple Criteria Decision Analysis Second Edition: State-of-the-Art Surveys, Springer, New York, pp. 817-850.

[21] Eyvindson, K., and Kangas, A. (2015). Using a Compromise Programming framework to integrating spatially specific preference information for forest management problems. Journal of MultiCriteria Decision Analysis, 22(1-2), 3-15.

[22] Gao, C., Lu, G., Yao, X., and Li, J. (2017). An iterative pseudo-gap enumeration approach for the Multidimensional Multiple-choice Knapsack Problem. European Journal of Operational Research, 260(1), $1-11$.

[23] Ghasemzadeh, F., and Archer, N. P. (2000). Project portfolio selection through decision support. Decision Support Systems, 29(1), 73-88.

[24] Greco, S., Ehrgott, M, and Figueira, J.R. (2016). Multiple Criteria Decision Analysis, Second Edition. Springer-Verlag, New York.

[25] Greco, S., Matarazzo, B., and Słowiński, R. (2001) Rough sets theory for multicriteria decision analysis. European Journal of Operational Research, 129(1) 1-47.

[26] Greco, S., Matarazzo, B., Słowiński, R. (2008). Dominance-based Rough Set Approach to Interactive Multiobjective Optimization. Chapter 5 [in]: Branke, J., Deb, K., Miettinen, K., Słowiński, R.(eds.), Multiobjective Optimization: Interactive and Evolutionary Approaches, LNCS 5252, State-of-the-Art Surveys, Springer, Berlin, pp. 121-155.

[27] Greco, S., Matarazzo, B., Słowiński, R. (2010). Dominance-based rough set approach to decision under uncertainty and time preference. Annals of Operations Research, 176(1), 41-75.

[28] Greco, S., Matarazzo, B., Słowiński, R. (2013). Beyond Markowitz with multiple criteria decision aiding. Journal of Business Economics, 83(1), 29-60.

[29] Greco, S., Matarazzo, B., Słowiński, R. (2016). Decision rule approach. Chapter 13 [in]: S. Greco, J. Figueira, and M. Ehrgott (eds.), Multiple Criteria Decision Analysis Second Edition: State-of-the-Art Surveys, Springer, New York, pp. 497-552.

[30] Greco, S., Matarazzo, B., Słowiński, R., Stefanowski, J. (2001). An algorithm for induction of decision rules consistent with dominance principle. [In]: W.Ziarko, Y.Yao (eds.): Rough Sets and Current Trends in Computing, LNAI 2005, Springer, Berlin, pp. 304-313. 
[31] Greiner, M., Fowler, J. W., Shunk, D. L., Carlyle, W. M., and McNutt, R. T. (2003). A hybrid approach using the analytic hierarchy process and integer programming to screen weapon systems projects. Engineering Management, IEEE Transactions on, 50(2), 192-203.

[32] Grushka-Cockayne, Y., Reyck, B. D., and Degraeve, Z. (2008). An integrated decision-making approach for improving European air traffic management. Management Science, 54(8), 1395-1409.

[33] Jaszkiewicz, A. (2002). On the performance of multiple-objective genetic local search on the 0/1 knapsack problem-a comparative experiment. Evolutionary Computation, IEEE Transactions on, 6(4), 402-412.

[34] Kadziński, M., Greco, S., Słowiński, R. (2014). Robust Ordinal Regression for Dominance-based Rough Set Approach to multiple criteria sorting. Information Sciences, 283(1), 211-228.

[35] Kadziński, M., Słowiński, R., Greco, S., (2015). Multiple criteria ranking and choice with all compatible minimal cover sets of decision rules. Knowledge-Based Systems, 89, 569-583.

[36] Kadziński, M., Słowiński, R., Greco, S. (2016). Robustness analysis for decision under uncertainty with rule-based preference model. Information Sciences, 328, 321-339.

[37] Kadziński, M., Tervonen, T., Tomczyk, M.K., Dekker, R. (2017). Evaluation of multi-objective optimization approaches for solving green supply chain design problems. Omega, 68, 168-184.

[38] Kadziński, M., Tomczyk, M.K., (2017). Interactive Evolutionary Multiple Objective Optimization for Group Decision Incorporating Value-based Preference Disaggregation Methods. Group Decision and Negotiation, https://doi.org/10.1007/s10726-016-9506-6

[39] Kangaspunta, J., Liesiö, J., and Salo, A. (2012). Cost-efficiency analysis of weapon system portfolios. European Journal of Operational Research, 223(1), 264-275.

[40] Karasakal, E., and Aker, P. (2016). A multicriteria sorting approach based on data envelopment analysis for R\&D project selection problem. Omega, https://doi.org/10.1016/j.omega.2016.12.006.

[41] Keeney, R. L. (1987). An analysis of the portfolio of sites to characterize for selecting a nuclear repository. Risk Analysis, 7(2), 195-218.

[42] Kiziltan, G., and Yucaoğlu, E. (1983). An algorithm for multiobjective zero-one linear programming. Management Science, 29(12), 1444-1453.

[43] Klamroth, K., and Wiecek, M. M. (2000). Dynamic programming approaches to the multiple criteria knapsack problem. Naval Research Logistics, 47(1), 57-76.

[44] Knoke, T., Paul, C., Hildebrandt, P., Calvas, B., Castro, L. M., Hartl, F., and Fernandez, G. F. C. (2016). Compositional diversity of rehabilitated tropical lands supports multiple ecosystem services and buffers uncertainties. Nature communications, 7, Article number: 11877. 
[45] Korotin, V., Popov, V., Tolokonsky, A., Islamov, R., and Ulchenkov, A. (2016). A multi-criteria approach to selecting an optimal portfolio of refinery upgrade projects under margin and tax regime uncertainty. Omega, https://doi.org/10.1016/j.omega.2016.11.003

[46] Liesiö, J. (2014). Measurable multiattribute value functions for portfolio decision analysis. Decision Analysis, 11(1), 1-20.

[47] Liesiö, J., Mild, P., and Salo, A. (2007). Preference programming for robust portfolio modeling and project selection. European Journal of Operational Research, 181(3), 1488-1505.

[48] Liesiö, J., Mild, P., and Salo, A. (2008). Robust portfolio modeling with incomplete cost information and project interdependencies. European Journal of Operational Research, 190(3), 679-695.

[49] Lourenço, J. C., Morton, A., and Costa, C. A. B. (2012). PROBE - A multicriteria decision support system for portfolio robustness evaluation. Decision support systems, 54(1), 534-550.

[50] Lourenço, J. C., Soares, J. O., and Bana e Costa, C. A. (2017). Portfolio robustness evaluation: a case study in the electricity sector. Technological and Economic Development of Economy, 23(1), 59-80.

[51] Marinakis, V., Doukas, H., Xidonas, P., and Zopounidis, C. (2017). Multicriteria decision support in local energy planning: An evaluation of alternative scenarios for the Sustainable Energy Action Plan. Omega, 69, 1-16.

[52] Markowitz, H. (1952). Portfolio selection. The journal of finance, 7(1), 77-91.

[53] Mavrotas, G., and Diakoulaki, D. (1998). A branch and bound algorithm for mixed zero-one multiple objective linear programming. European Journal of Operational Research, 107(3), 530-541.

[54] Mavrotas, G., Figueira, J. R., and Siskos, E. (2015). Robustness analysis methodology for multiobjective combinatorial optimization problems and application to project selection. Omega, 52, 142-155.

[55] Mavrotas, G., and Florios, K. (2013). An improved version of the augmented -constraint method (AUGMECON2) for finding the exact pareto set in multi-objective integer programming problems. Applied Mathematics and Computation, 219(18), 9652-9669.

[56] Miettinen, K., Hakanen, J., and Podkopaev, D. (2016). Interactive Nonlinear Multiobjective Optimization Methods. [in]: S. Greco, J. Figueira, and M. Ehrgott (eds.), Multiple Criteria Decision Analysis Second Edition: State-of-the-Art Surveys, Springer, New York, pp. 927-976.

[57] Miettinen, K., Ruiz, F., and Wierzbicki, A.P. (2008). Introduction to multiobjective optimization: interactive approaches. Multiobjective Optimization. Springer Berlin Heidelberg, pp. 27-57.

[58] Mild, P., Liesiö, J., and Salo, A. (2015). Selecting Infrastructure Maintenance Projects with Robust Portfolio Modeling. Decision Support Systems, 77, 21-30. 
[59] Montibeller, G., Franco, L. A., Lord, E., and Iglesias, A. (2009). Structuring resource allocation decisions: A framework for building multi-criteria portfolio models with area-grouped options. European Journal of Operational Research, 199(3), 846-856.

[60] Morton, A., Keisler, J. M., and Salo, A. (2016). Multicriteria portfolio decision analysis for project selection. Chapter 28 [in]: S. Greco, J. Figueira, and M. Ehrgott (eds.), Multiple Criteria Decision Analysis Second Edition: State-of-the-Art Surveys, Springer, New York, pp. 1269-1298.

[61] Pawlak, Z. (1991). Rough Sets. Theoretical Aspects of Reasoning about Data. Kluwer, Dordrecht.

[62] Pendharkar, P. C. (2014). A decision-making framework for justifying a portfolio of IT projects. International Journal of Project Management, 32(4), 625-639.

[63] Romero, C. (2001). Extended lexicographic goal programming: a unifying approach. Omega, 29(1), 63-71.

[64] Rong, A., and Figueira, J. R. (2013). A reduction dynamic programming algorithm for the bi-objective integer knapsack problem. European Journal of Operational Research, 231(2), 299-313.

[65] Salo, A., Keisler, J., and Morton, A. (Eds.). (2011). Portfolio decision analysis: improved methods for resource allocation (Vol. 162). Springer Science \& Business Media.

[66] Słowiński, R., Greco, S., Matarazzo, B. (2014). Rough-Set-Based Decision Support. Chapter 19 [in]: E.K. Burke and G. Kendall (eds.): Search Methodologies: Introductory Tutorials in Optimization and Decision Support Techniques, 2nd edition, Springer, New York, pp. 557-609.

[67] Słowiński, R., Greco, S., Matarazzo, B. (2015). Rough Set Methodology for Decision Aiding. Chapter 22 [in]: J.Kacprzyk and W.Pedrycz (eds.): Handbook of Computational Intelligence, Springer, Berlin, pp. 349-370.

[68] Stefanowski, J. (1998). On rough set based approaches to induction of decision rules. [in]: L.Polkowski and A. Skowron (eds.): Rough Sets in Data Mining and Knowledge Discovery. Vol.1, Physica-Verlag, Heidelberg, pp. 500-529.

[69] Stummer, C., and Heidenberger, K. (2003). Interactive RandD portfolio analysis with project interdependencies and time profiles of multiple objectives. Engineering Management, IEEE Transactions on, 50(2), 175-183.

[70] Susmaga, R., Słowiński, R., Greco, S., Matarazzo, B. (2000). Generation of reducts and rules in multiattribute and multi-criteria classification. Control and Cybernetics, 29, 969-988.

[71] Yunes, T. H., Napolitano, D., Scheller-Wolf, A., and Tayur, S. (2007). Building efficient product portfolios at John Deere and Company. Operations Research, 55(4), 615-629. 
[72] Tavana, M., Khalili-Damghani, K., and Abtahi, A. R. (2013). A fuzzy multidimensional multiple-choice knapsack model for project portfolio selection using an evolutionary algorithm. Annals of Operations Research, 206(1), 449-483.

[73] Tsamboulas, D., Yiotis, G. S., and Panou, K. D. (1999). Use of multicriteria methods for assessment of transport projects. Journal of Transportation Engineering, 125(5), 407-414.

[74] Ulungu, E. L., Teghem, J., and Ost, C. (1998). Efficiency of interactive multi-objective simulated annealing through a case study. Journal of the Operational Research Society, 49(10), 1044-1050.

[75] Vetschera, R., and De Almeida, A. T. (2012). A PROMETHEE-based approach to portfolio selection problems. Computers \& Operations Research, 39(5), 1010-1020.

[76] Vianna, D. S., and Arroyo, J. E. C. (2004). A GRASP algorithm for the multi-objective knapsack problem. Computer Science Society, 2004. SCCC 2004. 24th International Conference of the Chilean, IEEE, pp. 69-75.

[77] Wang, J., and Hwang, W. L. (2007). A fuzzy set approach for R\&D portfolio selection using a real options valuation model. Omega, 35(3), 247-257.

[78] Willis, C. E., and Perlack, R. D. (1980). A comparison of generating techniques and goal programming for public investment, multiple objective decision making. American Journal of Agricultural Economics, $62(1), 66-74$.

[79] Zheng, J., Cailloux, O., and Mousseau, V. (2011). Constrained multicriteria sorting method applied to portfolio selection. [in]: International Conference on Algorithmic DecisionTheory, Springer Berlin Heidelberg, pp. 331-343

[80] Zhu, Q., Dou, Y., and Sarkis, J. (2010). A portfolio-based analysis for green supplier management using the analytical network process. Supply Chain Management: An International Journal, 15(4), 306-319. 